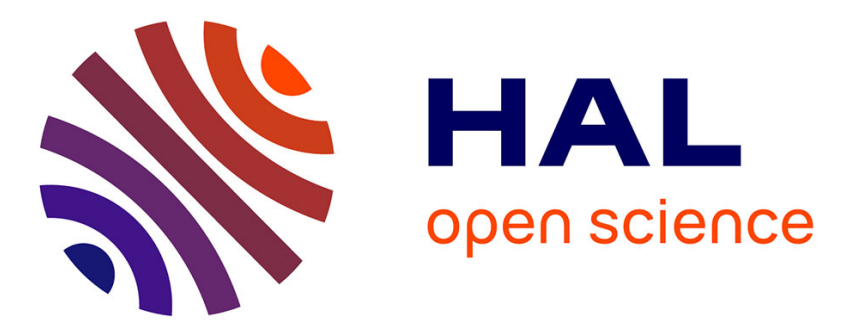

\title{
A Probabilistic Model Based Optimization for Aircraft Scheduling in Terminal Area under Uncertainty
}

\author{
Ying Huo, Daniel Delahaye, Mohammed Sbihi
}

\section{To cite this version:}

Ying Huo, Daniel Delahaye, Mohammed Sbihi. A Probabilistic Model Based Optimization for Aircraft Scheduling in Terminal Area under Uncertainty. Transportation research. Part C, Emerging technologies, 2021, 10.1016/j.trc.2021.103374 . hal-03342823

\section{HAL Id: hal-03342823 \\ https://hal-enac.archives-ouvertes.fr/hal-03342823}

Submitted on 13 Sep 2021

HAL is a multi-disciplinary open access archive for the deposit and dissemination of scientific research documents, whether they are published or not. The documents may come from teaching and research institutions in France or abroad, or from public or private research centers.
L'archive ouverte pluridisciplinaire HAL, est destinée au dépôt et à la diffusion de documents scientifiques de niveau recherche, publiés ou non, émanant des établissements d'enseignement et de recherche français ou étrangers, des laboratoires publics ou privés. 


\title{
A Probabilistic Model Based Optimization for Aircraft Scheduling in Terminal Area under Uncertainty
}

\author{
Ying $\mathrm{HUO}^{\mathrm{a}, *}$, Daniel Delahaye ${ }^{\mathrm{a}}$, Mohammed SBIHI ${ }^{\mathrm{a}}$ \\ ${ }^{a}$ ENAC, Université de Toulouse, 7 Avenue Edouard Belin, Toulouse 31055, France
}

\begin{abstract}
Air traffic management relies strongly on the accuracy of prediction, while uncertainties such as weather, navigation accuracy, pilot operations, etc. may weaken the performance of predictive tools and cause potential safety issues or reduced capacity. Among all controlled airspace, the Terminal Maneuvering Area (TMA) is one of the most complex areas in which flight safety can be easily affected by unpredictable disturbances. This paper addresses an aircraft scheduling problem under uncertainty with the aim of providing a robust schedule for arrival flights. Uncertainty quantification and propagation along the routes are realized in a trajectory model that formulates the time information as random variables. Conflict detection and resolution are performed at waypoints of a predefined network based on the predicted time information from the trajectory model. By minimizing the expected number of conflicts, consecutively operated flights can be well separated. Apart from the proposed model, two other models - the deterministic model and a model that incorporates separation buffers - are presented as benchmarks. A meta-heuristic simulated annealing algorithm combined with a time decomposition sliding window is proposed for solving a case study of the Paris Charles de Gaulle (CDG) airport. Further, a simulation framework based on the Monte-Carlo approach is implemented so as to evaluate the optimized solutions obtained from the three models. Statistical comparison among the results shows instability of the model that incorporates the separation buffers, in contrast, the proposed model has absolute advantages in both stability and the conflict absorbing ability when uncertainty arises.
\end{abstract}

Keywords: Aircraft scheduling problem, arrival management, uncertainty, probabilistic theory, simulated annealing

\footnotetext{
${ }^{*}$ Corresponding author

Email addresses: ying.huo@recherche.enac.fr (Ying HUO), delahaye@recherche.enac.fr (Daniel Delahaye), sbihi@recherche.enac.fr (Mohammed SBIHI)
} 


\section{Introduction}

The sudden outbreak of the COVID-19 pandemic throughout the world has led to a dramatic loss in air transportation. By April 2020, the number of global flights dropped by nearly $80 \%$, with international flights being the most affected [1]. With concerted efforts from the governments and international organizations, such as the International Civil Aviation Organization (ICAO) and the World Health Organization (WHO), the air transport industry has gradually recovered, starting with inner-continental operations [2]. It is clear that even though the impact of the pandemic on the aviation industry will continue for several years and the predicted growth of air traffic will be realized with a delay [3, 4, air transportation will continue to gradually reestablish, in conjunction with aligned industry guidance and the development of increasingly effective medical treatment. When mainstream travel returns, air traffic development, air traffic efficiency and safety will remain a critical issue to be considered. In the domain of air traffic management, one of the areas of high complexity is the Terminal Maneuvering Area (TMA). As the area where all arrivals converge, the safety issue is more impactful than other segments during aircraft operation. As we know, the variations of aircraft trajectory due to uncertainties could lead to potential conflicts, since the system that assists the decision-making process for air traffic controllers takes scant consideration of such perturbations. Consequently, air traffic controllers must intervene in flight operations according to their experience and intuition, which further increases their workload and further influences operational efficiency.

The potential effects of uncertainty have been noticed by departments in charge of air traffic management. In Europe, Single European Sky ATM Research (SESAR) has specified the interest of advancing air traffic services in terms of prediction accuracy, taking into account the intrinsic uncertainty in the arrival segment [5. Related projects have been carried out to improve trajectory prediction such as COPTRA and TBOMET, and more recently, a new project named START was launched to ensure the safety of air traffic while enhancing resilience when disturbances arise [6]. In this context, we believe that future systems are required to take the prediction error into account, thus arrival aircraft scheduling in TMA needs to simultaneously incorporate multiple considerations, such as uncertainty, safety constraints, and efficiency.

In this paper, we propose a novel method to determine a robust arrival schedule that can potentially improve the ability to hedge against uncertainties during flight operation while still satisfying the various constraints required for safety. Conflict detection and resolution are performed taking into account the prediction errors on the nominal aircraft trajectories.

The paper is organized as follows: A summary of related research is covered in Section 2 Section 3 describes the model formulations including the proposed model and two other models that serve as benchmarks. In accordance with the characteristics of each model, different objective functions are given for the proposed model and the benchmarks, respectively. Section 4 introduces the solution approach that we use to tackle our problem. Then, in Section 5 a simulation framework is introduced to investigate the performances of the presented models under disturbances. In Section 6 , the computational results are illustrated, and the simulation results obtained based on the optimized solutions from the three models are compared in terms of conflict absorption ability when uncertainty arises. Finally, Section 7 concludes the paper.

\section{State of the art}

The literature related to our work covers several aspects. First, our problem is essentially an aircraft scheduling problem for the arrival segment. Second, this problem is enhanced by taking the uncertainty into account. Aircraft sequencing and scheduling problems are broadly studied for the runway and the terminal area. As we know, the runway is the main bottleneck of the airport system, therefore the runway scheduling problem is intensively targeted. [7, 8, have conducted a series of studies starting from one single runway case with a mixed-integer zero-one formulation, extending to multi-runway cases. The solution approach evolves accordingly from the linear programming to the heuristic-based approach. One of the practical issues that affects the implementation of the arrival schedule is the Position Shifting Constraints (PSC) 9]. This constraint has been used in a great many works to improve traffic efficiency under the restriction of the first-come-first-serve (FCFS) policy taking the constraints of separation requirements into consideration. This idea has consequently spread and has been implemented in the research for sequencing and scheduling 
problems with multiple solution algorithms such as genetic algorithm 10 and dynamic programming [11. Further research incorporating the trade-off between contradictory objectives such as delay, throughput and operation costs, etc. start to gain more interest [12. Many works have considered the arrival sequencing and scheduling problem as a job shop scheduling problem where the runways or the route segments represent machines and aircraft considered to be jobs with predefined sequences [13, 14, 15.

Air traffic management can not only benefit from the change of flight schedule, but also from the building of innovative techniques for the arrival system. One of the most effective achievements is the development of the Point Merge System (PMS) by EUROCONTROL. Point merge replaces the traditional route structure with a more flexible one, consisting of a point (the merge point) and predefined legs that are equidistant from the merge point. Flights on the legs are directed to the merge point at appropriate times. From this design, some delays can be absorbed and an optimal sequence can be built. Moreover, the continuous descent operations can be integrated into this technique, thus flights in the terminal area are efficiently operated [16, 17, 18].

The above-mentioned studies are optimized or simulated based on exact information. However, in real operations, arrival sequencing and scheduling are carried out in a dynamic environment, where the predicted flight information and operational conditions in the airspace or the airport vary with time. Many works are dedicated to better understanding the pattern of prediction error in flight operation [19, while as a general term for all kinds of errors, the statistical attributes of the uncertainties being analyzed are highly dependent on the information available within multiple facets, such as airspace configuration, weather conditions, traffic density, or human factors. [19] used the Flight Update Message (FUM) that indicates the expected arrival time of flights at waypoints to estimate the prediction errors. In the work of [20], the prediction error of sector capacity evaluation is estimated. The predicted demand estimated in each sector is provided by the Enhanced Traffic Management System (ETMS), and the actual history of flight information is used as the reference for deriving the prediction error. 21] investigated the wind forecast error and aircraft performance error based on the information provided by the decision support tool. All of these works demonstrate that uncertainty is a critical issue inherent to air traffic management that must be considered.

Typical approaches used for uncertainty optimization include stochastic programming, robust optimization, and chance-constrained optimization [22, 23]. The most common applications of stochastic programming are the two-stage or multi-stage stochastic problems [24, 25, where one assumes that the uncertain parameters can be represented by the known probability distribution based on complete knowledge of the uncertainty. The selection of the tackled problems for the stages are usually based on the prioritization of the particular information required. In other words, for a two-stage problem, variables provide prior information before the realization of uncertainty parameters have been decided in the first stage; further, corrective measures (or recourse) are activated at the second stage to mitigate effects from uncertainty and to avoid infeasibilities. The general process is to take decisions for the first stage, such that the total cost of the first stage and the expected cost of the second stage are minimized. Due to uncertainty, the cost of the second stage can be considered as a random variable, and the expected function is introduced for the second stage. One broadly used method is the Sample Average Approximation (SAA) method, which approximates the expectation function by averaging the values obtained from a finite number of scenarios. In [26, the implementation and performance of the SAA method based on the Monte-Carlo approach has been comprehensively studied from both theoretical and experimental aspects for a stochastic optimization problem. The accuracy of stochastic optimization is based on the expansion of scenario exploration, while as the problem size gets bigger, the number of random variables increases, which drastically yields increased scenarios. Consequently, it is computationally intensive to adapt this approach to a high dimensional problem.

In robust optimization, the probability distribution of the parameter representing the underlying uncertainty is normally unknown. The concept of robust optimization is to define the reasonable uncertainty set, within which the optimized solution is computed [27]. Robust optimization is a conservative method that focuses on immunizing against the worst-case scenario derived from all the possible input data [27, 28. One of the common uses of robust optimization in dealing with the aircraft sequencing and scheduling problem is the robust min-max regret method. This method attempts to minimize the maximum regret value (the difference of objective values between a made decision and the optimal decision) obtained from the worst scenario 29. 30 implemented both the robust optimization model and the stochastic optimization model 
for the runway scheduling problem and provided an explicit comparison between these two models in terms of model formulation and performance of the results. According to different problems and designs, chance constrained optimization can sometimes be classified into the field of stochastic programming [31 or robust optimization [32. As the name indicates, chance constrained optimization allows for the violation of some inequalities by introducing a desired probability level 33. In 34, a chance constrained optimization approach is established by including probabilistic sector capacity constraints for the large scale air traffic flow management problem. With this method, a less conservative solution can be obtained compared to the robust optimization approach while maintaining a certain level of solution quality. Among multiple forms of robust optimization and stochastic programming, interpreting the uncertainty to probability related parameters sometimes is necessary, which indicates the wide implementation of probabilistic modeling. An abundance of studies focus on the air traffic flow management problem in the enroute segment by applying probabilistic interpretation for the time information and then the expected number of flights are estimated for specific sectors 35, 36, 37. Uncertainty can also be managed by imposing separation buffers between the consecutively operated aircraft [38, 39]. By tackling a similar conflict detection and resolution problem in the Paris CDG airport, 40, 41 addressed the uncertainty by enlarging the separation minima under a simulation optimization framework in order to reduce the effect of uncertainty in terms of conflicts. Intuitively speaking, inserting separation buffers with respect to the nominal separation minima is a very effective way to generate a robust solution. However, regarding the growth of traffic demand, fixed buffering limits the airspace capacity, reduces the flexibility of aircraft scheduling, and therefore introduces a more complex problem. The approach proposed here is developed based on the deterministic optimization model proposed in 42 . The model framework is retained, whereas several adjustments are made to better adapt to optimization under uncertainty. First, tailored separation time requirements have been computed for aircraft that pass through the same link/node. Second, the uncertainty has been incorporated into this model by introducing random variables for the uncertain time information of the predicted aircraft trajectories. By assuming the random variables are normally distributed, a single-stage stochastic problem is established with an objective function computing the expected number of conflicts in the whole network.

Additionally, for the purpose of comparison, two other problems are taken from previous works and serve as benchmarks. The first is the deterministic problem that does not take the uncertainty into consideration. The second refers to [40, in which the uncertainty is managed by using a buffer increasing approach. This approach tries to mitigate potential separation loss due to uncertainty by increasing the nominal required separation by a fixed amount of time.

Another issue that needs to be considered after the optimization of the problem is the evaluation of the solution performance. For example, in the work of [43, an airport stand assignment problem is constructed that takes the environmental impact and schedule disruptions into account. A simulation model was built to analyze the optimized solution under multiple close-to-reality scenarios so as to explore the situations that are of greatest interest. Motivated by the optimization and simulation combined framework, we proposed a two-step framework to include the problem optimization and the simulation-based solution evaluation. The contributions of this work are summarized as follows:

- A novel probabilistic optimization model taking the uncertainty into account for the arrival aircraft scheduling problem is proposed. Uncertainty is managed by introducing the random variables to represent the temporal information on the waypoints of the aircraft arrival routes. Regarding the realization of the uncertainty, the propagation feature is taken into account based on the real flight operation. The model formulation is then combined with the Markov assumption and probability theory. By taking advantage of the properties of normal distribution, an analytical evaluation of interactions between aircraft in terms of the conflict measurement is obtained. The objective function is then set with the expected number of conflicts occurring in the network, which enables an adjustment of the separation between aircraft pairs by considering the available temporal and spatial resources in the airport's surrounding area. In our study, the uncertainty is considered as an integrated element and reflected by the probability distribution of random variables, which increases the compatibility of this model to real-world application.

- Based on a consideration that the further the prediction is from the current time, the higher the uncer- 
tainties will be, an uncertainty propagation model is applied by incorporating the time decomposition and sliding window frame, where the window starting time means the recent flight information update in this window, and this time is regularly shifted to comply with the real world situation. Moreover, the time window limits the uncertainty propagation in a reasonable range.

- A simulation environment that introduces various kinds of disruptions is built to evaluate the qualities of solutions from the proposed model. Except for the proposed model, two other models will be used for comparison: a deterministic model and a model incorporating separation buffers. Comparing the simulation results among the three models shows the reliability of the performance of the proposed model.

\section{Problem description and mathematical modeling}

\subsection{Problem overview}

Flights that are intended to land at an airport are required to arrive at the designated entry points of the surrounding airspace of this airport at a specific time and then get into the TMA following the Standard Terminal Arrival Route (STAR). Flight flows from multiple directions merge at certain points to be sequenced and then proceed to the designated runways. During this process, the schedule should be carefully determined according to the predefined constraints or parameters for flights such as separation requirements, the runway acceptance rate, and the unexpected uncertainty. Focusing on the above issues, we address the arrival scheduling problem in the TMA area and runways under the uncertainty so as to provide a robust solution that can avoid potential risks.

\subsection{Network Structure}

Similar to the previous work of [44, we model the TMA arrival network as a graph $\mathcal{G}(\mathcal{N}, \mathcal{L})$. The node set $\mathcal{N}$ is the set of waypoints and the link set $\mathcal{L}$ is the set of the links interconnecting these waypoints with straight-line segments. We assume that $\mathcal{N}=\mathcal{N}_{e} \cup \mathcal{N}_{w} \cup \mathcal{N}_{r}$ where $\mathcal{N}_{e}$ contains the entry points of the TMA, $\mathcal{N}_{r}$ represents the runway thresholds, and $\mathcal{N}_{w}$ is the set of waypoints used to connect the entry points to the runway thresholds. This graph concisely represents the physical environment (e.g., Standard Terminal Arrival Route (STAR)), in a discretized manner with information such as locations of the metering points, distances and altitudes. For each pair $\left(\nu_{e}, \nu_{r}\right) \in \mathcal{N}_{e} \times \mathcal{N}_{r}$ there exists one route $\left(\nu_{e}, \nu_{1}, \ldots, \nu_{m}, \nu_{r}\right)$ in the graph $\mathcal{G}$ connecting the entry point $\nu_{e}$ to the runway threshold $\nu_{r}$. Each aircraft follows exactly one of these routes corresponding to its entry point and its assigned runway. Fig 1 displays a model example of a route network: the Paris CDG airport where we have four entry points $\mathcal{N}_{e}=\{$ MOPAR, LORNI, OKIPA, BANOX $\}$ and two runways $\mathcal{N}_{r}=\{27 \mathrm{R}, 26 \mathrm{~L}\} . \mathcal{N}_{w}$ is composed of 10 nodes and $\mathcal{L}$ of 18 links. In this example, each TMA entry point corresponds to two routes specified by different runway assignments.

\subsection{Given data}

Assume that we are given a set of landing flights (or aircraft) $\mathcal{F}$, for $f \in \mathcal{F}$ the following data are given:

- $e_{f} \in \mathcal{N}_{e}:$ Entry point at the TMA;

- $t_{f}^{o}$ : TMA entry time (Flight arrival time at $e_{f}$ );

- $v_{f}^{o}$ : Entering speed at the TMA;

- $c_{f}$ : Wake turbulence category;

- $r_{f}^{o}$ : Initial assigned landing runway.

When we simulate the flight trajectory in the TMA, a possible speed profile should be provided. By referring to the real ground speed profile from the radar data, a continuously decelerated speed profile is implemented. In this case, the speeds of flights are decreasing with a constant deceleration rate until the Final Arrival Fix (FAF), the final speeds are defined as $110 \mathrm{kt}, 130 \mathrm{kt}$, and $150 \mathrm{kt}$ for aircraft with the categories heavy, medium and small, respectively. 


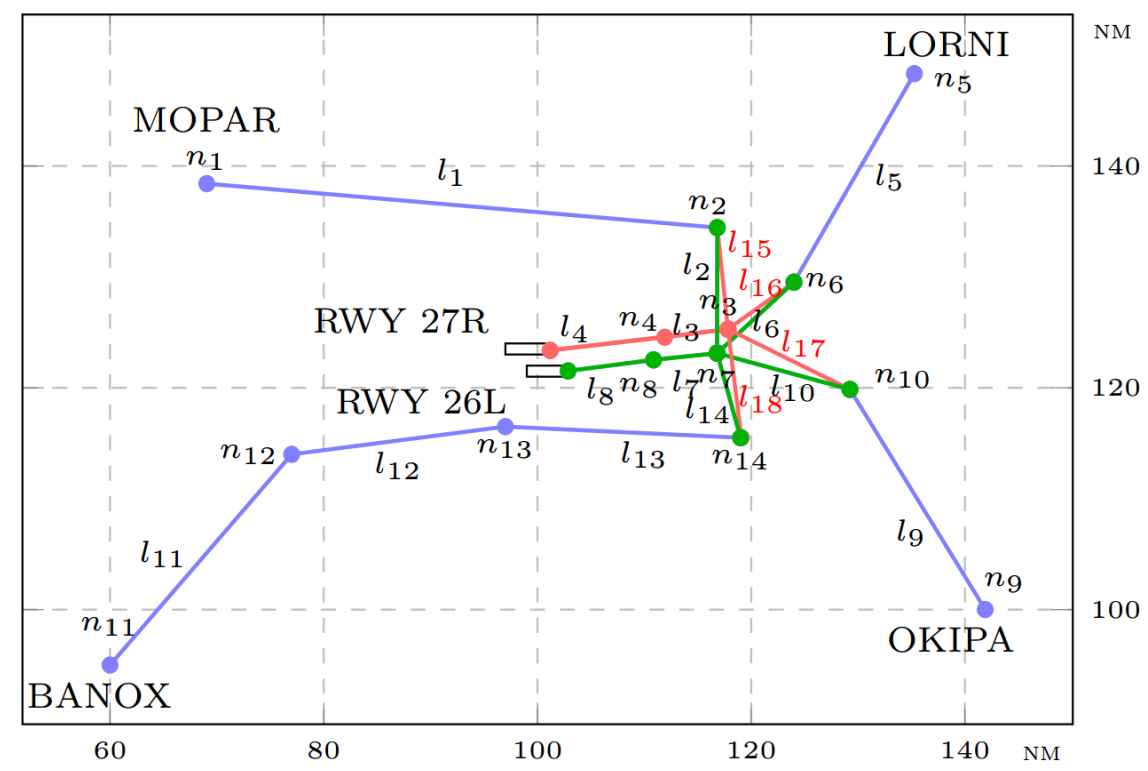

Figure 1: Arrival route structure of Paris CDG Airport in west configuration represented by network abstraction.

\subsection{Optimization models}

The optimization model is established to refer to the deterministic model proposed in 42 . The conflict detection method is modified to adapt to the presence of uncertainty.

\subsubsection{Decision variables}

To be in line with the flight operational environment during approach, we propose three kinds of decision variables for each flight $f \in \mathcal{F}$ with specific decision ranges:

- $t_{f}$ : the assigned TMA entry time of flight $f$, is adjusted by the number of time slots $\Delta t$ (typically the slot $\Delta t=5 s$ ). In order to shift the TMA entry time, we can either delay it or speed it up during the en-route phase. The maximum earliness and tardiness with respect to the initial TMA entry time is denoted as $t_{f}^{\min }$ and $t_{f}^{\max }$, respectively. In practice, delay is always more frequent than the earliness due to perturbations such as weather, safety margin, human factors etc., thus we have $\left|t_{f}^{\max }\right| \geq\left|t_{f}^{\min }\right|$. In this study, $t_{f}^{\max }$ and $t_{f}^{\min }$ are set to be 20 mins and -5 mins, respectively. More formally, we have:

$$
t_{f} \in T_{f}:=\left\{t_{f}^{o}+j \Delta t \mid j \in \mathbb{Z} \text { and } t_{f}^{\min } \leq j \Delta t \leq t_{f}^{\max }\right\}
$$

- $v_{f}$ : the assigned TMA entering speed of flight $f$, is selected by involving a user-defined speed increment $\Delta_{f}^{v}$ with respect to the initial TMA entry speed. $v_{f}^{\min }$ and $v_{f}^{\max }$ are given input data corresponding to the minimum and maximum allowable speeds for flight $f$. In this study, we set $v_{f}^{\min }=0.9 v_{f}^{o}$, $v_{f}^{\max }=1.1 v_{f}^{o}$ and $\Delta_{f}^{v}=0.01 v_{f}^{o}$. The TMA entry speed is given as:

$$
v_{f} \in V_{f}:=\left\{v_{f}^{o}+j \Delta_{f}^{v} \mid j \in \mathbb{Z} \text { and } v_{f}^{\min } \leq j \Delta_{f}^{v} \leq v_{f}^{\max }\right\}
$$

- $r_{f}$ : the assigned runway to flight $f$, which is chosen from the runway set:

$$
r_{f} \in \mathcal{N}_{r}
$$


Table 1: Wake turbulence separation minima for different categories of aircraft $s_{f, g}$, with associated aircraft categories, in NM.

\begin{tabular}{c|c|c|c|c}
\hline \multicolumn{2}{c|}{ Categories } & \multicolumn{3}{|c}{ Leading Aircraft $f$} \\
\cline { 3 - 5 } \multicolumn{2}{c}{} & Heavy & Medium & Light \\
\hline \multirow{2}{*}{$\begin{array}{c}\text { Trailing } \\
\text { Aircraft, } g\end{array}$} & Heavy & 4 & 3 & 3 \\
\cline { 2 - 5 } & Medium & 5 & 3 & 3 \\
\cline { 2 - 5 } & Light & 6 & 5 & 3 \\
\hline
\end{tabular}

Table 2: Single runway separation requirements, $\tau_{f, g}^{r c}$, with associated aircraft categories, in seconds.

\begin{tabular}{c|c|c|c|c}
\hline \multicolumn{2}{c|}{ Categories } & \multicolumn{3}{c}{ Leading Aircraft $f$} \\
\cline { 3 - 5 } \multicolumn{2}{c}{} & Heavy & Medium & Light \\
\hline \multirow{2}{*}{$\begin{array}{c}\text { Trailing } \\
\text { Aircraft, } g\end{array}$} & Heavy & 96 & 60 & 60 \\
\cline { 2 - 5 } & Medium & 157 & 69 & 69 \\
\cline { 2 - 5 } & Light & 207 & 123 & 82 \\
\hline
\end{tabular}

The decision vector associated with an arbitrary flight $f$ is denoted as $\mathbf{x}_{f}=\left(t_{f}, v_{f}, r_{f}\right)$ and the whole decision vector is denoted as $\mathbf{x}$. By assumption, there exists a unique route $R_{f}(\mathbf{x})$ connecting the entry point $e_{f}$ and the runway threshold of $r_{f}$. Moreover, using the speed profile of $f$ we can predict the arrival time at each node of the route $R_{f}(\mathbf{x})$. Let us denote the arrival time corresponding to the $i$-th node along its arrival route of $R_{f}(\mathbf{x})$ as $t_{f, i}(\mathbf{x})$, or simply $t_{f, i}$ in the sequel. Depending on the context, if no sequential requirement is put forward, we will also explicitly indicate the concerned node as $\nu$, where the arrival time will be represented as $t_{f, \nu}(\mathbf{x})$ or simply $t_{f, \nu}$.

\subsubsection{Separation rules and conflict detection}

To ensure flight safety, three separation requirements are considered: wake turbulence constraints, horizontal separation, and runway separation.

- Wake turbulence separation: the separation between two successively operated aircraft $f$ and $g$ should satisfy the wake turbulence separation minima $s_{f, g}$ associated with their aircraft categories $c_{f}, c_{g}$. The minimum separation standards are given in Table. 1.

- Horizontal separation: Aircraft must satisfy a minimum horizontal separation $h=3 N M$ based on the radar separation in the TMA.

- Runway separation: The runway separation requirements specified for two aircraft landing at the same runway with respect to their associated aircraft categories are given in Table. 2 [45. This requirement is also related to the associated occupancy time of aircraft on the runway.

A violation of the aforementioned separation rules gives rise to three types of conflicts. A link conflict refers to a violation of minimum wake turbulence separation between two successive aircraft flying on the same link. Catch-up conflict and overtake conflicts are examples of such conflict. A node conflict refers to the loss of horizontal separation between two aircraft on two different links adjacent to the same node. Finally, a runway conflict refers to the loss of runway separation between two aircraft landing at the same runway.

In order to adapt to a time-based metering system, which is convenient for conflict detection and metering the flows, the following lemmas transfer the distance-based separation to a time-based separation. Their proofs are presented later in Appendix A. Let $(f, g)$ be a pair of distinct aircraft operated consecutively.

Lemma 1. Let $\nu$ be a node belonging to the routes of $f$ and $g$. Assume that aircraft $f$ passes node $\nu$ before $g$. Then, there is no horizontal conflict between $f$ and $g$ at node $\nu$ if and only if:

$$
t_{g, \nu}-t_{f, \nu} \geq \tau_{f, g, \nu}^{n c}:=\left\{\begin{array}{l}
a, \quad \text { if } f \text { and } g \text { enter } \nu \text { through the same link (see Fig. 2a), } \\
\max (a, b), \quad \text { if } f \text { and } g \text { enter } \nu \text { from different links (see Fig. 2b). }
\end{array}\right.
$$


where

$$
\begin{aligned}
& a= \begin{cases}\frac{h}{v_{g, \nu}}, & \text { if } v_{g, \nu} \leq v_{f, \nu} \cos \theta, \\
\frac{h}{v_{f, \nu}}, & \text { if } v_{f, \nu} \leq v_{g, \nu} \cos \theta, \\
\frac{h \sqrt{\left(v_{f, \nu}^{2}+v_{g, \nu}^{2}-2 v_{f, \nu} v_{g, \nu} \cos \theta\right)}}{v_{g, \nu} v_{f, \nu} \sin \theta}, & \text { otherwise }\end{cases} \\
& b= \begin{cases}\frac{h}{v_{g, \nu},} & \text { if } v_{f, \nu} \cos \varphi \leq v_{g, \nu}, \\
\frac{h \sqrt{\left(v_{f, \nu}^{2}+v_{g, \nu}^{2}-2 v_{f, \nu} v_{g, \nu} \cos \varphi\right)}}{v_{g, \nu} v_{f, \nu} \sin \varphi}, & \text { otherwise }\end{cases}
\end{aligned}
$$

The angles $\theta$ and $\varphi$ are shown in Fig. 2. Moreover, $v_{f, \nu}, v_{g, \nu}$ denote the speeds of aircraft $f$ and $g$, respectively, at node $\nu$.

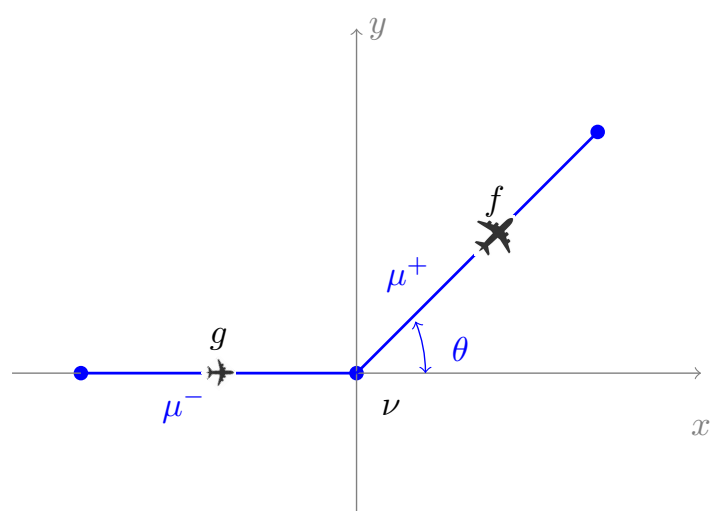

(a) Aircraft $f$ and $g$ arrive at node $\nu$ through the same (b) Aircraft $f$ and $g$ converge to node $\nu$ from different link.

Figure 2: Node conflict detection scenarios regarding different trajectory interaction patterns of the flight pair.

Lemma 2. Let $\mu=(\nu, \omega)$ be a link belonging to the routes of $f$ and $g$. Assume that aircraft $f$ enters the link $\mu$ before $g$ as shown in Fig. 3. The average speeds on $\mu$ for both aircraft are computed and denoted as $v_{f, \mu}$ and $v_{g, \mu}$. Then there is no conflict between $f$ and $g$ on link $\mu$ if and only if:

$$
t_{g, \nu}-t_{f, \nu} \geq \tau_{f, g, \mu}^{l c}:=\max \left(\frac{s_{f, g}}{v_{f, \mu}}, \frac{s_{f, g}}{v_{g, \mu}}+\frac{l\left(v_{g, \mu}-v_{f, \mu}\right)}{v_{g, \mu} v_{f, \mu}}\right)
$$

where $l$ represents the length of the link $\mu$.

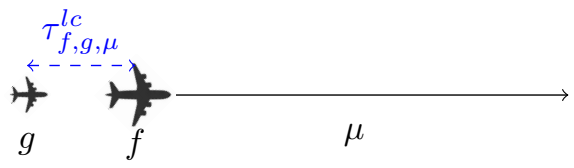

Figure 3: Link conflict detection configuration for two aircraft entering the same link consecutively.

Lemma 3. If $f$ and $g$ land at the same runway $r$ and $f$ lands before $g$, then there is no runway conflict between $f$ and $g$ if and only if:

$$
t_{g, r}-t_{f, r} \geq \tau_{f, g}^{r c}
$$

where $\tau_{f, g}^{r c}$ is given in Tab. 2 determined by the associated aircraft categories of aircraft $f$ and $g$. 
Based on the above mentioned lemmas, we introduce the following conflict indicators for each pair of aircraft $(f, g)$.

- Node conflict indicator

$$
\phi_{f, g, \nu}^{\mathrm{nc}}= \begin{cases}1, & \text { if } 0 \leq t_{g, v}-t_{f, \nu} \leq \tau_{f, g, \nu}^{n c} \text { or } 0 \leq t_{f, \nu}-t_{g, \nu} \leq \tau_{g, f, \nu}^{n c}, \quad \forall \nu \in R_{f}(\mathbf{x}) \cap R_{g}(\mathbf{x}) \cap \mathcal{N}_{w} \\ 0, & \text { otherwise }\end{cases}
$$

- Link conflict indicator,

$$
\phi_{f, g, \mu}^{\mathrm{lc}}=\left\{\begin{array}{l}
1, \text { if } 0 \leq t_{g, \nu}-t_{f, \nu} \leq \tau_{f, g, \mu}^{l c} \text { or } 0 \leq t_{f, \nu}-t_{g, \nu} \leq \tau_{g, f, \mu}^{l c}, \quad \forall \mu \in R_{f}(\mathbf{x}) \cap R_{g}(\mathbf{x}) \cap \mathcal{L}, \\
0, \text { otherwise }
\end{array}\right.
$$

- Runway conflict indicator

$$
\phi_{f, g}^{\mathrm{rc}}= \begin{cases}1, & \text { if } 0 \leq t_{g, r}-t_{f, r} \leq \tau_{f, g}^{r c} \text { or } 0 \leq t_{f, r}-t_{g, r} \leq \tau_{g, f}^{r c}, \quad \text { if } r_{f}=r_{g}=r \in \mathcal{N}_{r}, \\ 0, & \text { otherwise }\end{cases}
$$

The abusing notation $R_{f}(\mathbf{x}) \cap R_{g}(\mathbf{x}) \cap \mathcal{N}_{w}$ (resp. $\left.R_{f}(\mathbf{x}) \cap R_{g}(\mathbf{x}) \cap \mathcal{L}\right)$ designates the set of common nodes (resp. links) between the routes of $f$ and $g$. It should be noted that when aircraft are diverged to different landing runways, vertical separation will be imposed. For example, in Fig. 1, the arrival routes in red guide the aircraft to runway $27 \mathrm{R}$ and the green ones show the routes for aircraft landing at 26L. The altitudes assigned for the aircraft landing at different runways satisfy the vertical separation requirement and the horizontal separation for aircraft with different altitudes are not considered.

\subsubsection{Objective function of the deterministic model}

The objective function of this deterministic model (DERM) minimizes the total number of link conflicts, node conflicts, and runway conflicts in the network.

$$
G_{d}(\mathbf{x}):=\sum_{\substack{f, g \in \mathcal{F}, g>f}} \sum_{\nu \in R_{f}(\mathbf{x}) \cap R_{g}(\mathbf{x}) \cap \mathcal{N}_{w}} \phi_{f, g, \nu}^{\mathrm{nc}}+\sum_{\substack{f, g \in \mathcal{F} \\ g>f}} \sum_{\mu \in R_{f}(\mathbf{x}) \cap R_{g}(\mathbf{x}) \cap \mathcal{L}} \phi_{f, g, \mu}^{\mathrm{lc}}+\sum_{\substack{f, g \in \mathcal{F}, g>f \mid r_{f}=r_{g}}}^{\mathrm{rc}} \phi_{f, g}^{\mathrm{rc}}
$$

The first term in the sum counts the number of node conflicts, the second term counts the number of link conflicts and the last one counts the number of runway conflicts. Condition $g>f$ prevents double counting the conflicts.

\subsubsection{Objective function for the probabilistic-based model under the presence of uncertainty}

To incorporate the impact of uncertainty into the model establishment, one typical way is to consider the exact information with randomness. Based on DERM, the probabilistic model (PROM) needs to bridge the conflict detection with the potential prediction error. A natural way is to consider the arrival time information of the flights at particular waypoints as random variables.

Let us replace the deterministic arrival time at node $\nu: t_{f, \nu}$ by a random variable $T_{f, \nu}$ with a probability density function $\psi_{f, \nu}$. In the same way, conflict indicators (5), (6), (7) become random variables indicating the occurrence of a conflict and are represented as $\Phi_{f, g, \nu}^{\mathrm{nc}}, \Phi_{f, g, \mu}^{\mathrm{lc}}, \Phi_{f, g}^{\mathrm{rc}}$, respectively. By replacing the relative 
terms in (8), the objective function can be updated as:

$$
\begin{aligned}
& G_{p}(\mathbf{x})=\sum_{\substack{f, g \in \mathcal{F}, g>f}} \sum_{\nu \in R_{f}(\mathbf{x}) \cap R_{g}(\mathbf{x}) \cap \mathcal{N}_{w}} P\left(\Phi_{f, g, \nu}^{\mathrm{nc}}=1\right)+\sum_{\substack{f, g \in \mathcal{F} \\
g>f}} \sum_{\mu \in R_{f}(\mathbf{x}) \cap R_{g}(\mathbf{x}) \cap \mathcal{L}} P\left(\Phi_{f, g, \mu}^{\mathrm{lc}}=1\right)+\sum_{\substack{f, g \in \mathcal{F}, g>f \mid r_{f}=r_{g}}} P\left(\Phi_{f, g}^{\mathrm{rc}}=1\right) \\
& =\sum_{\substack{f, g \in \mathcal{F} \\
g>f}} \sum_{\nu \in R_{f}(\mathbf{x}) \cap R_{g}(\mathbf{x}) \cap \mathcal{N}_{w}} E\left(\Phi_{f, g, \nu}^{\mathrm{nc}}\right)+\sum_{\substack{f, g \in \mathcal{F} \\
g>f}} \sum_{\mu \in R_{f}(\mathbf{x}) \cap R_{g}(\mathbf{x}) \cap \mathcal{L}} E\left(\Phi_{f, g, \mu}^{\mathrm{lc}}\right)+\sum_{\substack{f, g \in \mathcal{F}, g>f \mid r_{f}=r_{g}}} E\left(\Phi_{f, g}^{\mathrm{rc}}\right) \\
& =E\left(\sum_{\substack{f, g \in \mathcal{F}, g>f}} \sum_{\nu \in R_{f}(\mathbf{x}) \cap R_{g}(\mathbf{x}) \cap \mathcal{N}_{w}} \Phi_{f, g, \nu}^{\mathrm{nc}}\right)+E\left(\sum_{\substack{f, g \in \mathcal{F}, g>f}} \sum_{\substack{\mu \in R_{f}(\mathbf{x}) \cap R_{g}(\mathbf{x}) \cap \mathcal{L} \\
\mu=(\nu, \omega)}} \Phi_{f, g, \mu}^{\mathrm{nc}}\right)+E\left(\sum_{\substack{f, g \in \mathcal{F}, g>f \mid r_{f}=r_{g}}} \Phi_{f, g}^{\mathrm{rc}}\right)
\end{aligned}
$$

where the three terms represent the expected number of conflicts on nodes, links, and runways.

In order to compute the objective of Eq. 9, the airborne uncertainty model must be specified. For the uncertainty model, there is no restriction in selecting the distribution for the random variable. A natural choice is a Gaussian distribution, which has been widely used [37, 38, 36, 46. We can also use more complex models such as a mixture of two distributions, where one of the distributions can be used to model the uncertainty for regular flights and the other to model flights with heavy delays. For the airborne uncertainty model, the chosen distribution must satisfy the flight constraint of Eq. 4.4 in the work of [47, which implies that the support of the density function, (where the support of the density function refers to the range of real numbers for which the density function is positive) is bounded. Suitable candidates for such properties are triangular and beta probability density functions. One example is the Program Evaluation and Review Technique (PERT), which is used in project management tools for characterizing the length of a task in a scheduling problem. The PERT distribution is defined as a Beta distribution with an assumption on the expected value regarding the minimum, most likely and maximum values of the variable and therefore provides a control on the probability assignment [48. Indeed, the accurate prediction of uncertainties along an entire aircraft's trajectory is not trivial. It is a function of space and time, which requires a mathematical expression for the uncertainty propagation mechanism along the route.

In our study, normal distribution is implemented for three reasons. First, the underlying computational characteristics of normal distribution are adapted to derive an analytical expression for the objective function of the proposed model. Second, in the absence of data to estimate the underlying distribution and by virtue of the central limit theorem, this choice seems reasonable. Third, the characteristics of normal distribution enable a unified expression of uncertain parameters, thus providing an analytical expression for conflict assessment. In fact, many other kinds of error distributions can be used, however, when no explicit representation of uncertainty propagation is available, the computational cost is expensive when exploring the scenarios using the repeated sampling method. Therefore, in this study, we assume that the prediction errors of aircraft arrival time at each waypoint $\nu$ (node) is approximately normally distributed, for which the variance is proposed to be proportional to the look-ahead time (the difference between the predicted and the current time, which corresponds to the time taken to make decisions). In our assumption, this means that the uncertainty is supposed to grow with respect to a reference time (current time). More precisely, for an arbitrary flight $f$, the random variable corresponding to the TMA entry time can be represented as:

$$
T_{f, 0} \sim \mathcal{N}\left(t_{f, 0}, \alpha\left(t_{f, 0}-t_{c}\right)\right)
$$

where $t_{c}$ denotes the current time, $t_{f, 0}$ denotes the predicted arrival time at the TMA entry node, $\alpha$ is a coefficient that defines the propagation rate of uncertainty, which is reflected in the variance of the probability distribution related to the look-ahead time. The uncertainties defined for the rest of the waypoints of flight $f$ are formulated based on Markov assumption, that is to say, the probability of the arrival time at the next point $\left(T_{f, i+1}\right)$ is independent on the past arrival time $\left(T_{f, 0}, \ldots, T_{f, i-1}\right)$, while it is conditionally related to the last arrival time $T_{f, i}$. We recall that in this notation, $T_{f, i}$ refers to the random variable representing the arrival time of the $i$-th waypoint on the arrival route of flight $f$. Thus the following relation is established:

$$
T_{f, i+1} \mid\left(T_{f, i}=t_{i}\right) \sim \mathcal{N}\left(t_{i}+\left(t_{f, i+1}-t_{f, i}\right), \alpha\left(t_{f, i+1}-t_{f, i}\right)\right)
$$


From this we can deduce that:

$$
T_{f, i} \sim \mathcal{N}\left(t_{f, i}, \alpha\left(t_{f, i}-t_{c}\right)\right)
$$

Then for the current setting of the uncertainty model, the components of Eq. 9 can be calculated by referring to the characteristics of normal distribution. For two aircraft $f$ and $g$ that overfly the same node $\nu$ consecutively, $T_{f, v}$ and $T_{g, \nu}$ are independent random variables, and the probability density function of the inter-arrival random variable $T_{g, \nu}-T_{f, \nu}$ is given by $\psi_{g, \nu} * \psi_{f, \nu}$, where $*$ is the convolution operator. We can infer that the inter-arrival time is also normally distributed:

$$
T_{g, \nu}-T_{f, \nu} \sim \mathcal{N}\left(t_{g, \nu}-t_{f, \nu}, \alpha\left(t_{g, \nu}+t_{f, \nu}-2 t_{c}\right)\right)
$$

Therefore, an analytical computation of the probabilities of the conflict that constitute the objective function (Eq. 9) can be conducted according the following steps:

$$
\begin{aligned}
P\left(\Phi_{f, g, \nu}^{\mathrm{nc}}=1\right) & =P\left(0 \leq T_{g, \nu}-T_{f, \nu} \leq \tau_{f, g, \nu}^{n c}\right)+P\left(0 \leq T_{f, \nu}-T_{g, \nu} \leq \tau_{g, f, \nu}^{n c}\right) \\
& =F_{f, g, \nu}\left(\tau_{f, g, \nu}^{n c}\right)-F_{f, g, \nu}(0)+F_{g, f, \nu}\left(\tau_{g, f, \nu}^{n c}\right)-F_{g, f, \nu}(0)
\end{aligned}
$$

where $F_{f, g, \nu}$ is the cumulative distribution function of the normal distribution associated with the interarrival random variable. Since the parameter $\alpha$ affects the variance of the random variable, it should be determined based on the time prediction error of airborne flights. Moreover, in the optimization, the value of $\alpha$ implicitly affects the overall separation assignment for the flight set. If $\alpha$ is set with a large value, an enlarged separation is required for each pair of flights to achieve small objective values. Empirically, $\alpha$ is set to 1 .

\subsubsection{Additional separation buffer based model}

Considering the model formulation, PROM is naturally expected to perform better than DERM when uncertainty arises. Therefore the approach that applies additional buffers proposed in the previous work of [40, is presented in our study and used as a comparative model. This model is denoted as BUFM and established on the basis of DERM by enlarging wake turbulence separation requirements, horizontal separation requirement and runway separation requirements with a given percentage $\kappa$. The value of $\kappa$ is chosen to be $20 \%$ which is a middle value that is used in 40. It is worth mentioning that the conflict detection model used for BUFM is the same as that used for DERM and PROM.

\section{Solution approach}

For problems related to arrival management, computational efficiency is essential. The heuristic algorithms are more likely to provide a fast and satisfying result, in which simulated annealing (SA) has been proven to be efficient and easy to be adapted to a complex problem that involves large-dimensional state space. Therefore, a time decomposition and sliding window approach combined with the simulated annealing algorithm that is similar to the one used in 42 is adapted for this study.

\subsection{Simulated annealing}

Simulated annealing is a meta-heuristic method to approximate global optimization in a large search space for an optimization problem [49. It is used to find an approximate global optimum in a fixed amount of time. The method involves heating and controlled cooling that mimics the process of metal annealing. An important control parameter in this optimization process is the temperature. The heating process explores the solution space to search for a temperature that can ensure a sufficient and deep exploration for the cooling process. The cooling process refers to the decreasing of the temperature which can be interpreted as a slow decreasing in the probability of accepting worse solutions while exploring the solution space. In SA, several factors should be specified for implementation: 
Table 3: Empirically-set parameter values of the simulated annealing algorithm with time decomposition approach.

\begin{tabular}{lc}
\hline Parameters & Values \\
\hline Geometrical temperature reduction coefficient & 0.99 \\
Number of iterations of each temperature step & 100 \\
Final temperature & $10^{-4} T_{0}$ \\
Time length of the sliding window & $2 \mathrm{~h}$ \\
Time shift of the sliding window & $1 \mathrm{~h}$ \\
\hline
\end{tabular}

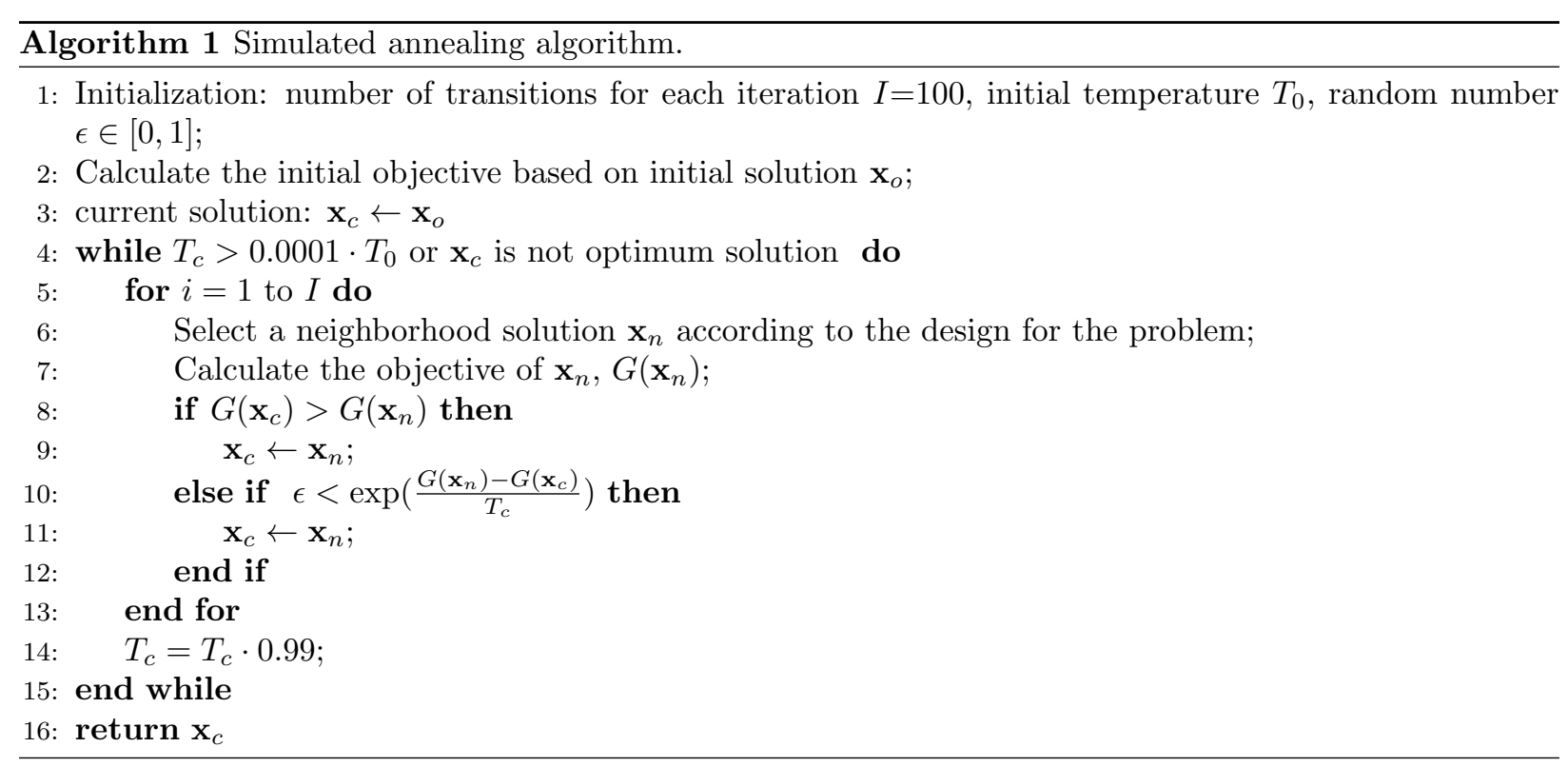

- The temperature is normally set to follow a geometric pattern and can be given as $T_{k}=\beta T_{k-1}$ for consecutive iterations, where $k$ denotes the finished number of iterations and $\beta$ is a cooling parameter which is set according to the problem.

- Neighborhood solution $\mathbf{x}_{n}$ is chosen from a design regarding the given circumstance. At each step, the quality of current solution $\mathbf{x}_{c}$ and the neighborhood solution are measured, and a decision is made whether or not to accept the neighborhood solution.

- Acceptance probability is generally specified with Metropolis criterion $e^{\frac{\Delta E}{T}}$, where $\Delta E$ is the difference of the objectives between the current solution and the neighborhood solution, and $T$ is the current temperature.

Following a repetition process of searching for neighborhood solutions and accepting the better solution, the algorithm will guide the process to a near optimal solution. The steps mentioned above are repeated until the temperature reaches a predefined minimum. The user-defined parameters of the algorithm are provided in Tab. 3. The parameters are mostly set through empirical analysis to ensure a suitable adaptation of the algorithm to our problem. The pseudo-code of the cooling procedure for the SA is given in Algorithm. 1 .

\subsection{Time decomposition and sliding window approach}

The time decomposition and sliding window approach is usually implemented with other optimization algorithms in order to divide the whole problem into sub-problems that belong to smaller time horizons to reduce the complexity of the overall problem. In this study, the time decomposition and sliding window frame also provides a realistic environment for the uncertainty propagation model in terms of updating 
the current time and limiting the look-ahead time in a reasonable range. Several parameters need to be introduced and specified for this approach.

- $T_{I}$ : starting time of the overall time horizon.

- $T_{E}$ : ending time of the overall time horizon.

- $L$ : time length of each sliding window.

- $Q$ : shifting time of the sliding window.

- $k$ : sequence number of the sliding window.

- $S_{k}$ : starting time of the $k$-th sliding window, where $S_{k}=T_{I}+Q(k-1)$.

- $E_{k}$ : ending time of the $k$-th sliding window, where $E_{k}=T_{I}+L+Q(k-1)$ and $E_{k} \leq T_{E}$.

As the name indicates, time windows are continuously sliding while solving the sub-problems. The window starts from an initial time $T_{I}$ and slides until the end of the window arrives at the ending time of the time horizon $T_{E}$.

\subsubsection{Flight status classification}

In order to determine the flights that are involved in a given time window, a classification rule for flight status is proposed. For each flight, the status is assigned based on its earliest possible time of entering the TMA and the latest possible time of landing on the runway. The total transition time $\Gamma_{f}$ of flight $f$ in the TMA is deterministically predicted by considering the lowest TMA entry speed and the longer arrival route. Necessary parameters for determining the flight status are:

- $\tau_{s}^{f}$ : the earliest possible TMA entry time of flight $f$, which is computed by $t_{f}^{o}-t_{f}^{\min }$.

- $\tau_{e}^{f}$ : the latest possible landing time of flight $f$, which is computed by $t_{f}^{o}+t_{f}^{\max }+\Gamma_{f}$.

Suppose that we optimize the $k$-th window, the flights will be classified and marked with a status according to the following rule:

- Completed flight: $\tau_{e}^{f}<S_{k}$, this status means that the flight has been optimized in a previous window and has no interaction with the flights that need to be optimized in the current window.

- Ongoing flight: $\tau_{s}^{f}<S_{k} \bigwedge \tau_{e}^{f}>S_{k}$, an ongoing flight means it has been optimized in the previous window, but it is still partially active in the network and may interact with flights that need to be optimized. Flights of this status are also included in the trajectory prediction but their decision variables are fixed.

- Active flight: $S_{k}<\tau_{s}^{f} \leq E_{k}$, the flights that need to be optimized in the current window are active flights.

- Planned flight: $\tau_{s}^{f}>E_{k}$, planned flights have their earliest TMA entry time located later than the ending time of the current window. As the window slides afterward, the planned flights will go through the statuses from active to ongoing and then completed.

For each window, only the flights with active or ongoing status will be involved in the sub-problem of the current window. 

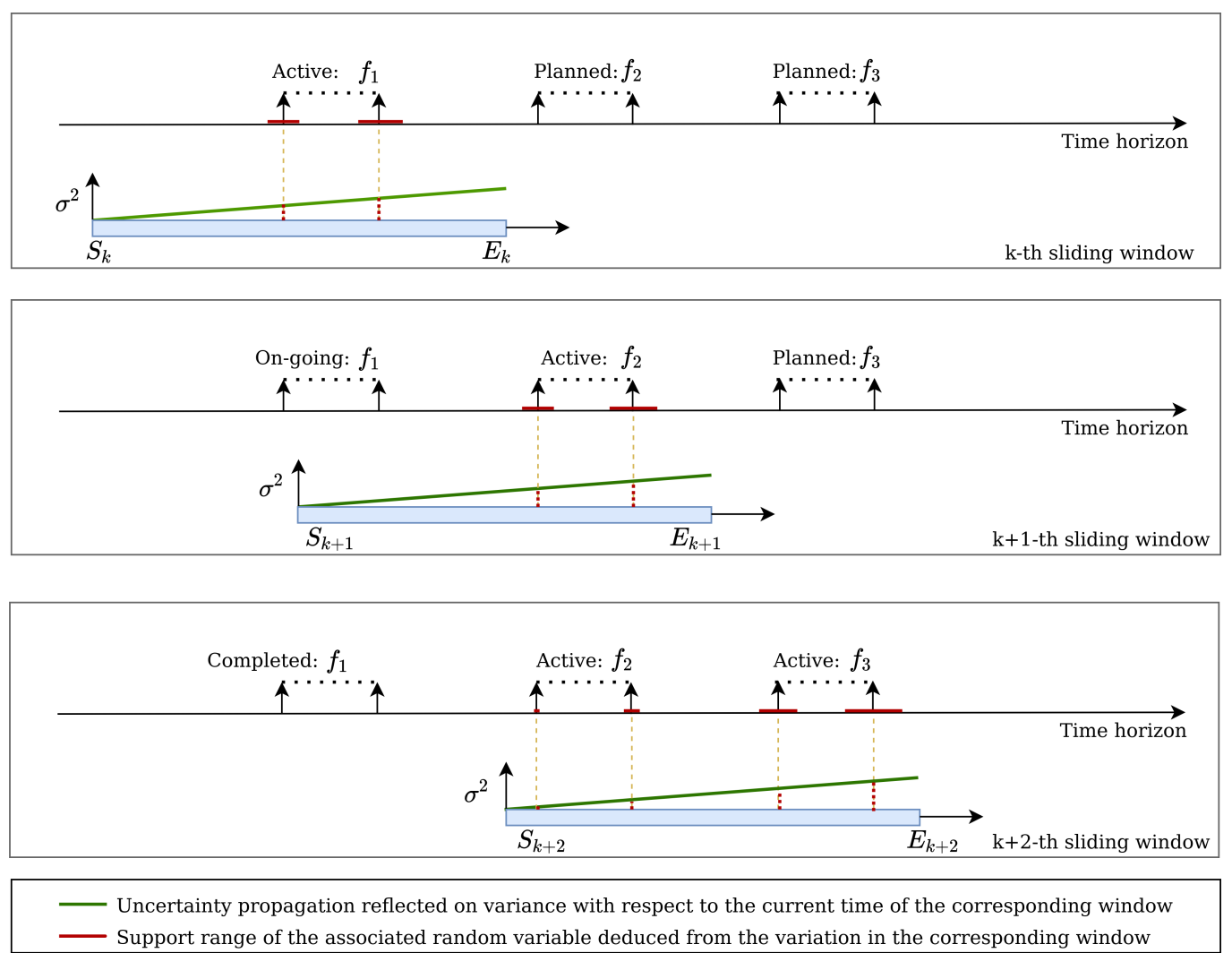

Figure 4: The uncertainty propagation for flights of different statuses under the sliding window frame.

\subsubsection{Uncertainty management under sliding window frame}

The time decomposition and sliding window frame enables a practical realization of uncertainty propagation model by providing regularly shifted current time $t_{c}$ and limited look-ahead time. The current time $t_{c}$ is set to be the time window beginning time. The length of the time window identifies the aircraft that will arrive in a reasonable range as it is not realistic to control arriving aircraft too far in advance, which puts a constraint on the look-ahead time to avoid an exaggerate increase of prediction error.

Fig. 4 conceptually illustrates how the random variables vary under the sliding window frame. In each of the figures, a coordinate is established, in which the blue stripe represents the time horizon of the current sliding window in the $x$-axis, the $y$-axis represents the possible values of the variance, and the green line associates the possible predicted times with values of variance for the corresponding random variables. For each flight, predicted TMA entry time (at the TMA entry node) and predicted landing time (at runway threshold) displayed by the black arrows are shown on the timeline. The red lines represent the associated variances. Three flights $f_{1}, f_{2}, f_{3}$ are displayed in the figure with their associated statuses of that window. As the probability distribution of a random variable is partially determined by the current time $t_{c}$, the probability distributions for the same flight on the same waypoint are different when the window slides to the next one.

The procedures of the sliding window frame considering the management of uncertainty are described in Algorithm 2, The neighborhood selection design for this problem is adjusted based on the one proposed in [42. In the original neighborhood function, the conflicts that a flight encounters are recorded as its cost. In order to increase the chance of selecting the aircraft with high costs, the roulette wheel selection method is used, in which the cost of each flight is proportional to its likelihood of being selected. The chosen flight from this approach will undergo a decision variable change. The advantage of this design is that the flights with higher costs are easier to be selected and the decision changes for those flights are more likely to be 


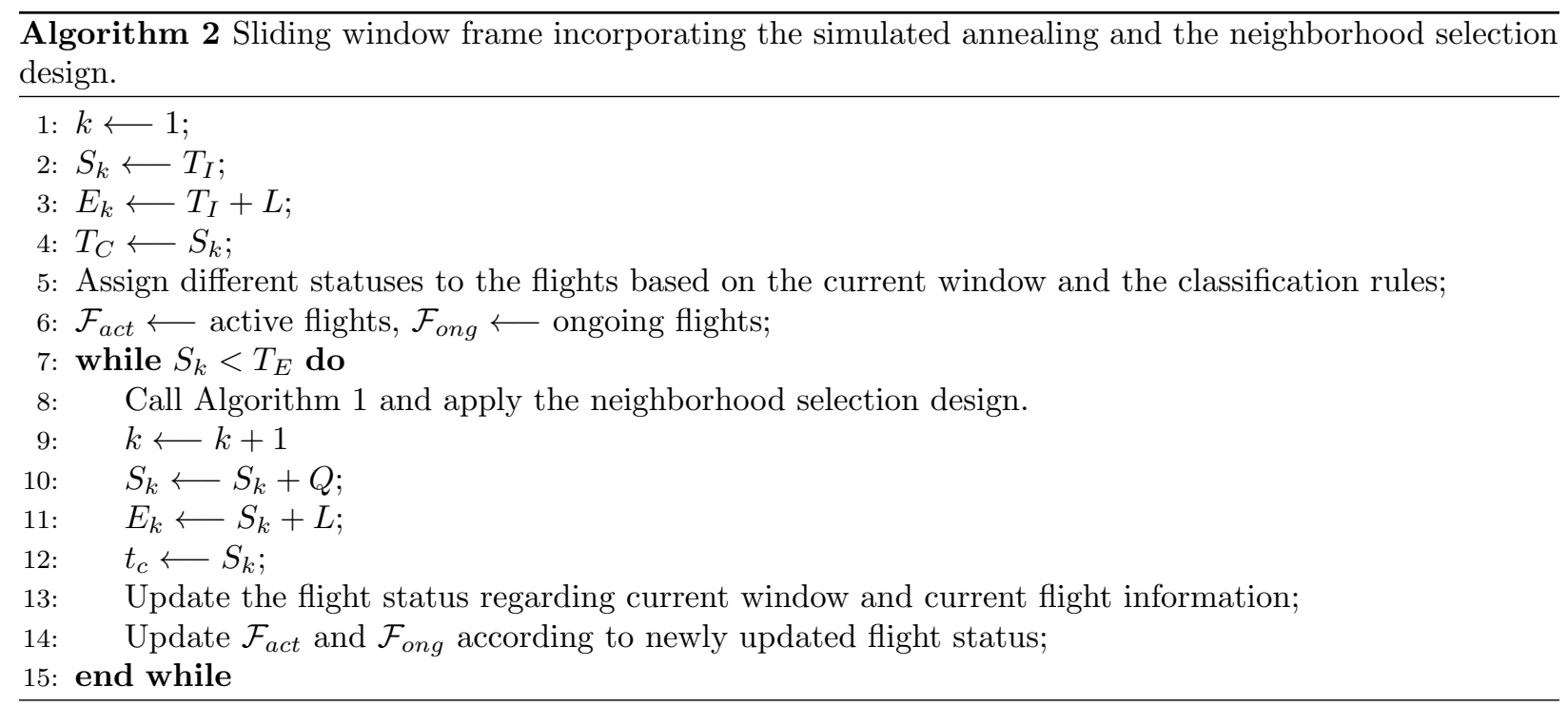

beneficial in minimizing the objective function. However, under the current design, only the flights whose costs are bigger than 0 can be selected. Considering the fact that all flights involved in optimization have potential effects on the overall performance of the model, we introduce a small value $\varsigma=0.0001$ to be added to all flights, regardless of their initial costs. Since $\varsigma$ is small, on the one hand, this change has no significant influence on the optimization efficiency. On the other hand, it allows the algorithm to exploit the solution space deeper since the decision variables of the flights with costs equal to 0 have the possibility of being changed. To solve PROM, the cost associated with a flight is the sum of the probabilities of conflict that it encounters in the whole network. It is worth mentioning that though both active and ongoing flights are involved in the problem, only the active flights are optimized with decision changes.

\section{Solutions evaluation}

In this section, we describe a simulation environment to test the optimized solutions obtained from the DERM, BUFM and PROM by means of stochastic perturbation.

\subsection{Simulation model for the solution evaluation}

The simulation model is established based on the Monte-Carlo approach. The main idea is to obtain a large number of outcomes by repeating the random perturbations on the optimized solutions so as to achieve the statistical analysis. We note that, in the process of evaluating the solution quality, the construction of a reliable simulation tool goes hand-in-hand with the development of the modeling frameworks for the optimization problem [31. The practical issue considered in PROM in line with the real-life situation is that for each flight, the arrival time of the next waypoint always depends on the arrival time at the current one. The sampling process in the simulation has taken this fact into account, thus the arrival times at all the passed waypoints of a flight are perturbed. Through sampling, each experiment will lead to a deterministic evaluation. The number of conflicts generated from each experiment is calculated and considered as a metric of the robustness. According to the law of large numbers, the number of replications is set to be 10000 to guarantee the accuracy of the statistical analysis.

\subsection{Simulation scenarios}

Considering how PROM and BUFM manage the uncertainty, two types of disturbance distribution are applied in the simulation model to evaluate the solution quality. 
- Normally distributed perturbation: in this case, the probability distributions of the random variables, where the samples are drawn from, are consistent with the random variables in the PROM as indicated in Eq. 10 and Eq. 11 for the waypoints along the route of a flight.

- Uniformly distributed perturbation: in this case, the random variables where the samples are drawn from follow uniform distribution. Their variances are identically computed as in the previous case: $\alpha\left(t_{f, 0}-t_{c}\right)$ on the TMA entry point and $\alpha\left(t_{f, i+1}-t_{f, i}\right)$ from the waypoint $i$ to the next one $i+1$. Mention that the variance of uniform distribution is defined as $(p-q)^{2} / 12$ where $p$ and $q$ are the maximum and minimum bound of this uniform distribution. Thus, the probability distribution for the random variables whose ranges defines the sample space can be specified by Eq. 15 and Eq 16 (in a form of $\mathcal{U}(p, q))$ :

$$
\begin{gathered}
T_{f, 0} \sim \mathcal{U}\left(t_{f, 0}-\sqrt{3 \alpha\left(t_{0}-t_{c}\right)}, t_{f, 0}+\sqrt{3 \alpha\left(t_{0}-t_{c}\right)}\right) \\
T_{f, i+1} \mid\left(T_{f, i}=t_{i}\right) \sim \mathcal{U}\left(t_{i}-t_{f, i}+t_{f, i+1}-\sqrt{3 \alpha\left(t_{f, i+1}-t_{f, i}\right)}, t_{i}-t_{f, i}+t_{f, i+1}+\sqrt{3 \alpha\left(t_{f, i+1}-t_{f, i}\right)}\right)
\end{gathered}
$$

In order to investigate the solution performance under different levels of uncertainty, two values of $\alpha$ are applied in the simulation. Thereby four scenarios are presented with combinations of probability distributions and levels of uncertainty, namely:

- Scenario $1(\mathrm{~N} 1)$ : samples are drawn from the domain of normally distributed random variables with a smaller variance by setting $\alpha=1$.

- Scenario 2 (N2): samples are drawn from the domain of normally distributed random variables with a higher variance by setting $\alpha=2$.

- Scenario 3 (U1): samples are drawn from the domain of uniformly distributed random variables with a smaller variance by setting $\alpha=1$.

- Scenario 4 (U2): samples are drawn from the domain of uniformly distributed random variables with a higher variance by setting $\alpha=2$.

\subsection{Time decomposition and sliding window frame for simulation}

In our simulation model for solution evaluation, the variance of the probability distribution associated with the flight arrival time at the TMA entry node is related to the current time $t_{c}(\mathrm{Eq}, 10)$. Since our data ranges from 0:00 to 24:00, it is clear that without the sliding window frame, the current time is fixed, hence the probability distributions of the random variables are unrealistic for some aircraft. Therefore, time decomposition and the sliding window frame is also applied in the simulation framework and the flight classification mechanism is applied for specifying the flights involved in the simulation for different time windows. In the simulation model, the length of the sliding window $L$ is set to 2 hours, and the whole day is divided into 12 windows, with the shift time $Q$ equals to 2 hours, as shown in Fig. 5 . The simulations are then conducted for each time window separately, where the status of a flight is classified based on the exact information provided in the optimized solutions associated to this flight.

The overall process of the simulation framework for the solution evaluation taking the sliding window frame into account is illustrated in Fig. 6 .

\section{Case study and results}

\subsection{Case study}

The actual arrival flight data on 18 February 2016 at the Paris CDG airport is applied to our model. There are two runway configurations at the Paris CDG airport: west-flow $(26 \mathrm{~L}, 27 \mathrm{R}-26 \mathrm{R}, 27 \mathrm{~L})$ and 


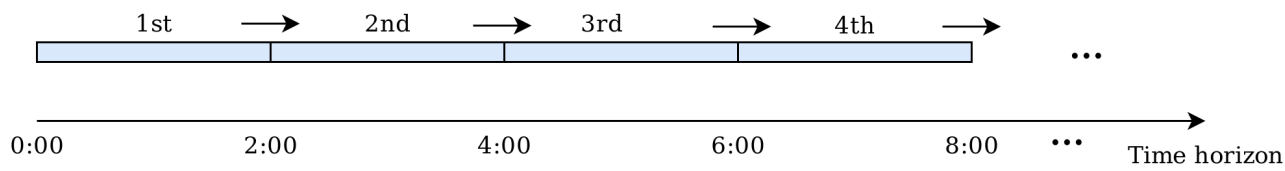

Figure 5: Sliding window frame applied in the simulation.

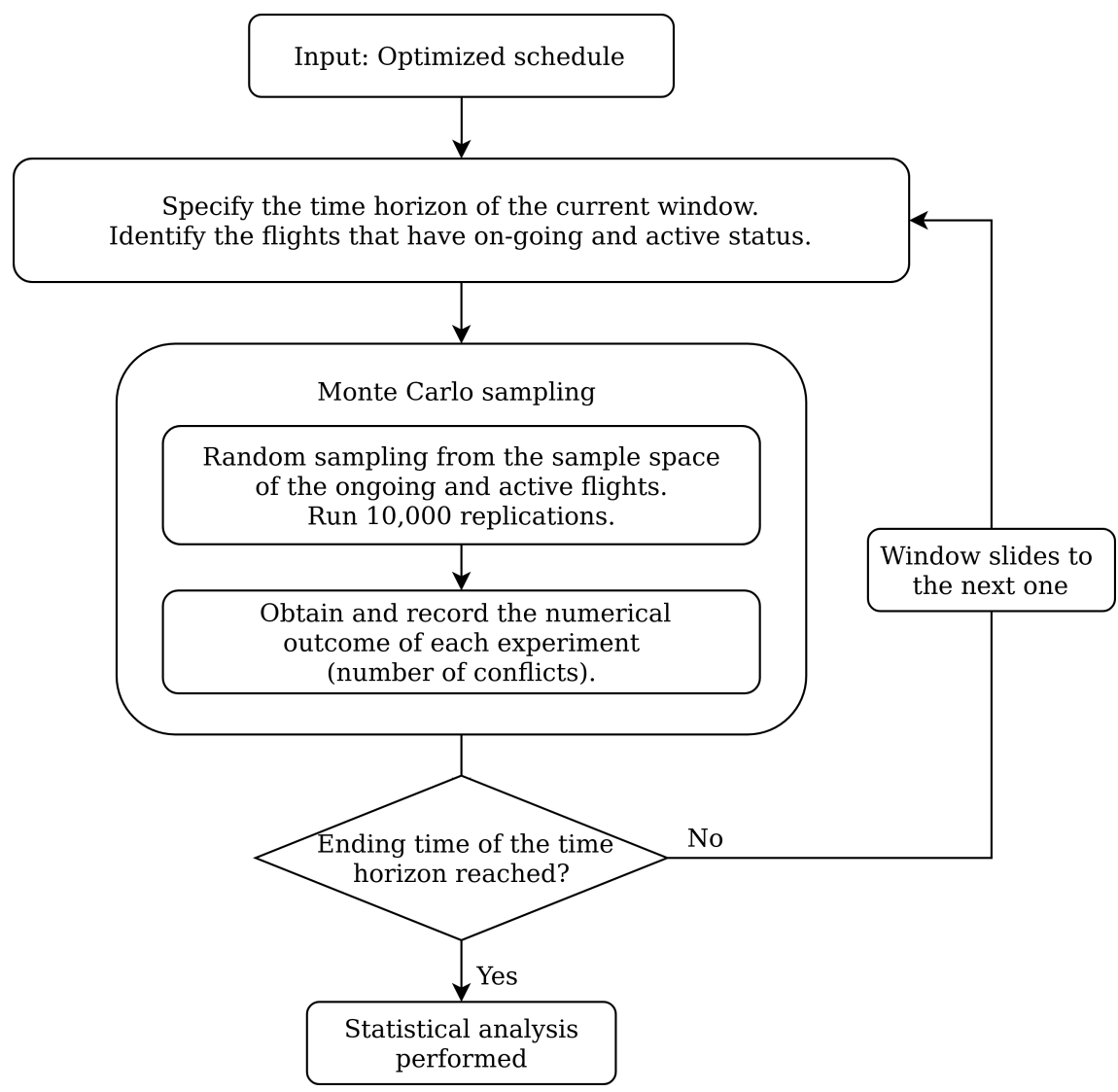

Figure 6: Overall process of the simulation framework for the solution evaluation.

east-flow (09L, 08R - 09R, 08L). This paper focuses on the more frequently used west-flow configuration. The TMA route structure of the Paris CDG is displayed in Fig. 1 .

The categories of operated flights are mostly large and medium. Tab. 4 provides the arrival flight information in the TMA. The overall optimization process is run on a $2.50 \mathrm{GHz}$ core i7 CPU, under a Linux operating system PC with Java code.

\subsection{Results for one window sub-problem}

Focusing on a single window allows a better comparison of the different models without the sliding effect. For example, in scenario N1, the sample space is consistent with the range of the random variables built in PROM model, therefore, we anticipate that the expected number of conflicts in the optimal solution of PROM is close to the average number of conflicts obtained from the simulation result of scenario N1.

In this single window case, the flights that are initially operated during the busy period from 6:00 to 8:00 are taken into account for our demonstration with respect to the optimization and solution evaluation processes. Associated information of arrival flights is given in Tab. 5 . In this period, we have 78 flights and all of them are active flights. 
Table 4: Traffic flow distribution of the flight data on 18 February 2016 at Paris CDG airport.

\begin{tabular}{cccc}
\hline & Medium & Heavy & Total \\
\hline MOPAR & $73(64.6 \%)$ & $40(35.4 \%)$ & 113 \\
LORNI & $153(80 \%)$ & $37(20 \%)$ & 190 \\
OKIPA & $146(79 \%)$ & $39(21 \%)$ & 185 \\
BANOX & $82(89 \%)$ & $10(11 \%)$ & 92 \\
\hline
\end{tabular}

Table 5: Information of the flights on 18th February 2016 between 6:00 to 8:00 in Paris CDG airport.

\begin{tabular}{cccc}
\hline & Medium & Heavy & Total \\
\hline MOPAR & 5 & 10 & 15 \\
LORNI & 21 & 3 & 24 \\
OKIPA & 21 & 2 & 23 \\
BANOX & 14 & 2 & 16 \\
\hline
\end{tabular}

\subsubsection{Optimization results for the single window case}

Considering the differences in the objective evaluations of the three models, the final results of the objective values have their own unique meanings. Tab. 6 displays the optimized objective values for the link, node and runway conflicts, for which the objective values of BUFM and DERM are 0. The results of DERM suggest that all aircraft can be well managed with required separation minima. With the separation requirement increased by $20 \%$, the objective function value of BUFM indicates that extra space between flight pairs is available under current traffic density, which further implies the potential of obtaining a robust solution. In contrast, the objective value of PROM that evaluates the expected number of conflicts is positive. This is because the uncertainty propagation model may yield random variables with big variances, therefore under limited time resources, the probability of conflict occurrence can not be eliminated.

The computational time of the optimization process is 30 seconds for PROM and 1.2 seconds for BUFM and DERM, respectively. Considering the window time horizon, they are all very efficient in solving the problems.

\subsubsection{Simulation-based solution evaluation for the single window case}

Once the optimization process is finished, the solutions are taken as inputs and randomly perturbed based on the predefined scenarios. The simulation results are displayed using statistical frequency distribution. Frequency here refers to the occurrence of a particular value of the conflict number in the network. Fig. 7 displays the comparison of frequency distributions for PROM, BUFM, DERM under four scenarios, in which we note that the distributions resemble a bell shape. Therefore, the statistical indicators of mean and standard deviation can provide a reference in the performance comparison. In the figure, no significant differences can be clearly observed for the standard deviation, while it is clear that PROM outperforms BUFM with less conflicts generated. For more detailed information, Tab. 7 aggregates the average conflicts arising in the simulation. In the last row, the percentage refers to the proportion of the average conflicts in PROM and BUFM with respect to that obtained from DERM. We can see that during a busy period, the solution obtained from PROM protects against $50 \%$ of the conflicts, while BUFM can only prevent less than $20 \%$ of conflicts based upon the average number of conflicts associated with DERM.

It should be noted that the demonstration of the single window case is supposed to verify the connections

Table 6: The final objective values of the optimization process for the window from 6:00-8:00

\begin{tabular}{ccccc}
\hline Objective value & Node & link & Runway & Total \\
\hline DERM & 0 & 0 & 0 & 0 \\
BUFM & 0 & 0 & 0 & 0 \\
PROM & 16.67 & 28.32 & 11.79 & 56.78 \\
\hline
\end{tabular}


between the PROM and the simulation model. As mentioned above, the final value of the objective function in PROM should be close to the simulation results obtained from scenarios N1. This can be observed by comparing the objective values in the last row of Tab. 6] and the simulation results in the fourth column in Tab. 7, where the differences are minor.

N1

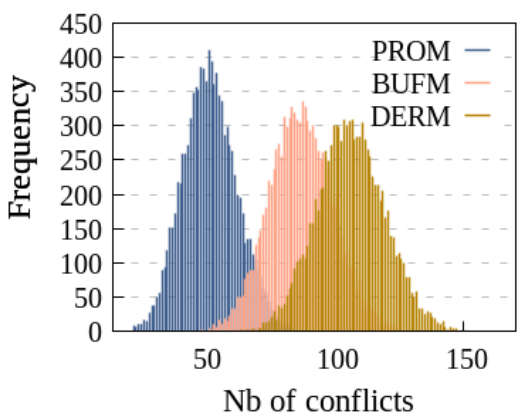

U1

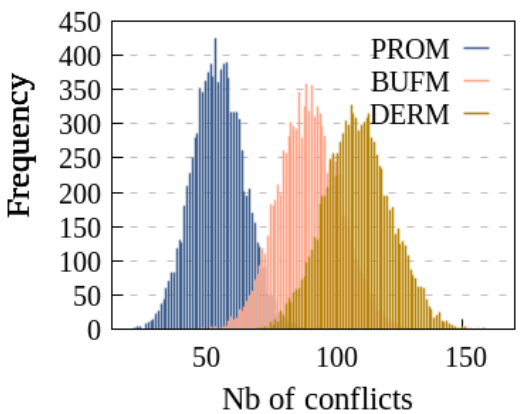

N2

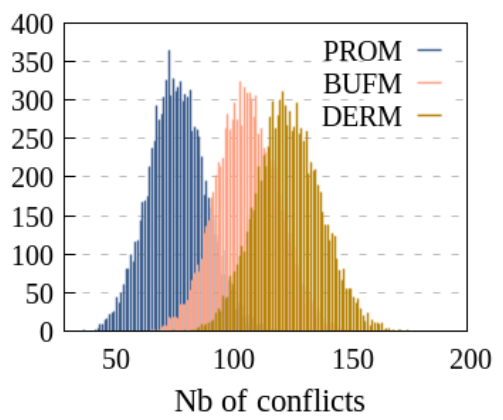

$\mathrm{U} 2$

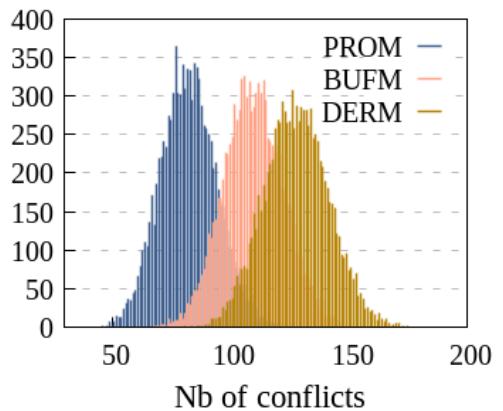

Figure 7: The frequency distributions of the simulation experiments in terms of number of conflicts. The simulation results are obtained from the Monte-Carlo simulation based on the optimized solutions of the PROM, BUFM, DERM during the time windows from 6:00 to 8:00.

\subsection{Results of optimization and solution evaluation for one day case}

In the previous investigation, it has been proven that PROM provides a better solution than BUFM in a high traffic density case. We continue to investigate the model performance in different problem sizes. For the optimization part, time horizon is enlarged and different problem scales are investigated based on the application of the sliding window frame. Once the optimization of the one day data is completely finished, the optimized solutions are then transferred to the solution evaluation process as inputs. Following the same steps as in the single window case, both the optimization results and the results for the solution evaluation are presented.

\subsubsection{Optimization results of the one day case}

Knowing that the air traffic densities are different from the sub-problems for the associated windows, in Tab. 8, the number of flights (ongoing and active) that define the sub-problem size and the associated final objective values are displayed. In addition, the execution times of associated sub-problems in the optimization process are also given for the 24 windows. As for DERM and BUFM, the objective values are all 0 for the sub-problems. The total computational time of BUFM and DERM are 5 seconds and 2 
Table 7: Simulation results of average numbers of conflicts in a single window for each resource based on the optimized solutions of PROM, BUFM, and DERM under four scenarios.

\begin{tabular}{|c|c|c|c|c|c|c|c|c|c|c|c|c|}
\hline \multicolumn{4}{|c|}{ N1 } & \multicolumn{3}{|c|}{$\mathrm{N} 2$} & \multicolumn{3}{|c|}{ U1 } & \multicolumn{3}{|c|}{ U2 } \\
\hline Model & DERM & BUFM & PROM & DERM & BUFM & PROM & DERM & BUFM & PROM & DERM & BUFM & PROM \\
\hline Link & 33.19 & 25.04 & 13.9 & 38.64 & 30.35 & 21.66 & 34.13 & 26.07 & 14.85 & 39.62 & 31.08 & 23.34 \\
\hline Node & 54.73 & 46.23 & 27.25 & 64.15 & 56.92 & 40.21 & 56.45 & 48.2 & 29.11 & 66 & 58.47 & 42.7 \\
\hline Runway & 18.68 & 16.13 & 11.2 & 21.91 & 19.71 & 15.67 & 19.19 & 16.69 & 11.96 & 22.33 & 20.29 & 16.3 \\
\hline Total & 106.6 & 87.41 & 52.35 & 124.69 & 107.08 & 77.54 & 109.8 & 90.97 & 55.92 & 127.97 & 109.84 & 82.42 \\
\hline Percentage & $100 \%$ & $81.9 \%$ & $49.1 \%$ & $100 \%$ & $\mathbf{8 5 . 9} \%$ & $62.2 \%$ & $100 \%$ & $82.9 \%$ & $50.9 \%$ & $100 \%$ & $85.8 \%$ & $64.4 \%$ \\
\hline
\end{tabular}

Table 8: Optimization results of PROM including the computational time for multiple problem sizes and the associated final objective values.

\begin{tabular}{cccccc}
\hline Time window & $\begin{array}{c}\text { Active } \\
\text { flights }\end{array}$ & $\begin{array}{c}\text { On-going } \\
\text { flights }\end{array}$ & Total & $\begin{array}{c}\text { Execution } \\
\text { time(s) }\end{array}$ & $\begin{array}{c}\text { Objective } \\
\text { value }\end{array}$ \\
\hline 0:00-2:00 & 2 & 0 & 2 & 0.03 & 0 \\
1:00-3:00 & 1 & 2 & 3 & 0.008 & 0 \\
$2: 00-4: 00$ & 3 & 1 & 4 & 0.026 & 0 \\
3:00-:500 & 28 & 1 & 29 & 4.327 & 0.08 \\
4:00-6:00 & 35 & 13 & 48 & 7.478 & 0.126 \\
5:00-7:00 & 39 & 16 & 54 & 13.388 & 3.35 \\
6:00-8:00 & 78 & 11 & 89 & 32.249 & 47.43 \\
$7: 00-9: 00$ & 71 & 40 & 111 & 40.643 & 60.01 \\
$8: 00-10: 00$ & 41 & 49 & 90 & 28.392 & 13.16 \\
9:00-11:00 & 57 & 16 & 53 & 26.19 & 12.09 \\
10:00-12:00 & 68 & 21 & 89 & 36.487 & 32.76 \\
11:00-13:00 & 33 & 44 & 77 & 20.23 & 2.31 \\
12:00-14:00 & 43 & 14 & 57 & 14.37 & 1.45 \\
13:00-15:00 & 39 & 20 & 59 & 14.164 & 0.53 \\
14:00-16:00 & 38 & 20 & 58 & 17.917 & 1.16 \\
15:00-17:00 & 55 & 20 & 75 & 25.366 & 17.395 \\
16:00-18:00 & 44 & 31 & 75 & 24.321 & 6.64 \\
17:00-19:00 & 53 & 23 & 76 & 25.484 & 10.57 \\
18:00-20:00 & 55 & 22 & 77 & 21.609 & 8.89 \\
19:00-21:00 & 38 & 34 & 72 & 19.179 & 0.614 \\
20:00-22:00 & 37 & 14 & 51 & 14.847 & 0.68 \\
21:00-23:00 & 22 & 23 & 45 & 2.89 & 0 \\
22:00-24:00 & 12 & 9 & 21 & 0.5 & 0 \\
\hline
\end{tabular}

seconds, respectively, which are both highly promising for solving a complex problem with a large volume of air traffic.

\subsubsection{Results of solution evaluation for one day case}

The realization of the sliding window framework leads to independent simulations of each window. In consequence, it is necessary to aggregate the results with a clear demonstration while displaying as much information as possible. Therefore, boxplot is selected to present the statistic features of the number of conflicts with a size of 10000 (number of replications) obtained from the simulation. In Fig. 8 four figures corresponding to the simulation results for $\mathrm{N} 1, \mathrm{~N} 2$, U1, and U2 from up to down are illustrated, in which the blue boxes represent the results of PROM, while the red and yellow boxes correspond to the results associated with BUFM and DERM, respectively. Overall, the plots in the four figures show the same variation trend in terms of distribution of conflicts on the time windows. By checking the number of flights 

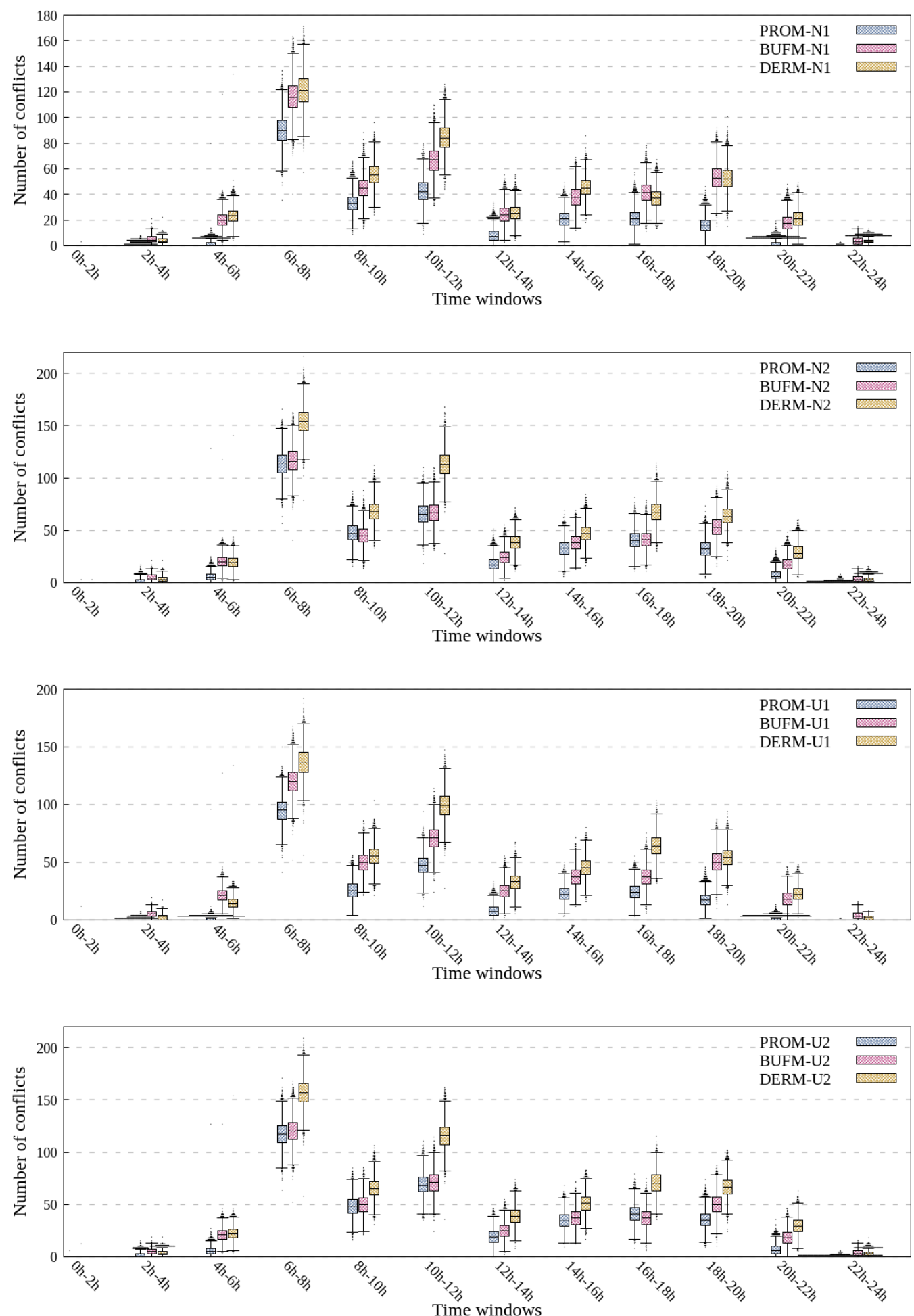

Figure 8: A comparison of simulation results of PROM, BUFM and DERM based on four scenarios presented by boxplot. 
Table 9: Solution performances indicators of PROM and BUFM represented by the percentage of conflicts absorbed based on the results of DERM for 12 windows and four scenarios.

\begin{tabular}{llcccccccccccc}
\hline & Time window & 1 & 2 & 3 & 4 & 5 & 6 & 7 & 8 & 9 & 10 & 11 & 12 \\
\hline \multirow{2}{*}{ N1 } & BUFM (\%) & $*$ & -55 & 14 & 4 & 19 & 21 & 5 & 16 & -11 & -1 & 17 & -22 \\
& PROM (\%) & $*$ & 90 & 95 & 26 & 40 & 50 & 69 & 54 & 42 & 69 & 93 & 100 \\
\hline \multirow{2}{*}{ N2 } & BUFM (\%) & $*$ & -64 & -39 & 10 & 8 & 23 & 15 & 4 & 23 & -3 & 4 & -95 \\
& PROM (\%) & $*$ & 57 & 73 & 26 & 30 & 42 & 55 & 30 & 40 & 49 & 75 & 96 \\
\hline \multirow{2}{*}{$\mathrm{U} 1$} & BUFM (\%) & $*$ & -92 & -43 & 12 & 9 & 28 & 24 & 18 & 42 & 7 & 19 & -140 \\
& PROM (\%) & $*$ & 93 & 94 & 31 & 53 & 53 & 75 & 50 & 62 & 68 & 95 & 100 \\
\hline \multirow{2}{*}{$\mathrm{U} 2$} & BUFM (\%) & $*$ & -63 & -28 & 9 & 1 & 21 & 14 & 4 & 26 & -9 & 16 & -98 \\
& PROM (\%) & $*$ & 53 & 74 & 25 & 26 & 41 & 51 & 33 & 42 & 47 & 76 & 98 \\
\hline
\end{tabular}

in the time windows, we can see that the number of active flights involved in the simulation has a significant impact on the number of conflicts generated for each model. We continue the comparison of the boxplot sets by focusing on the median values. It is obvious that PROM corresponds to the least number of conflicts compared to the results associated with BUFM and DERM for all of the time windows in the four scenarios. Comparing the results between BUFM and DERM, occasionally, BUFM has higher median values than DERM, which mostly appear in the case when there are not enough flights involved. This phenomenon suggests that the approach of inserting buffer is not practically reliable in reducing the potential risk caused by uncertainty.

In order to evaluate the quality of the solutions numerically, the solution performance indicator is evaluated as: $\left(C_{\text {DERM }}-C_{\mathrm{X}}\right) / C_{\mathrm{DERM}} \cdot 100$, where $C_{\mathrm{DERM}}$ is the total number of conflicts obtained from the simulation of DERM, and $C_{\mathrm{X}}$ can be either total number of conflicts associated with BUFM or PROM from the simulation. This indicator essentially reflects the robustness of the solutions obtained from the models that take uncertainty into account with respect to the deterministic model in terms of conflict absorption percentage. In Tab. 9, the performance indicator associated with PROM and BUFM are given. In the first time window, $*$ represents 0 conflict. The negative values are observed in some cases of BUFM, which is basically consistent with the cases shown in Fig. 8 where the median values of BUFM is higher than that of DERM.

We then investigate the variation of the performance indicators in Tab. 9 in contrast with the number of active flights. Considering the indicators associated with PROM (the 3rd, 5th, 7th and 9th row in Tab. 9p, in all scenarios, high values appear in the time windows with only a small number of active flights (2nd and 12 th time window) and their adjacent time windows (3rd, 11th time window). When the number of active flights increases, the performances slightly drop (5th, 6th, 7th, 8th, 9th, 10th time windows). For the busiest time window (4th time window), $25 \%-31 \%$ of conflict absorption is achieved. However, the performance of the BUFM shows no such pattern. When we focus on the perturbation with different levels of variance (N1 v.s N2 and U1 v.s U2), PROM shows a reduction in its performance in the scenario with a higher variance. However, BUFM shows no clear sign of how the performance indicators change. Moreover, except for the negative performance indicator, the highest performance indicator of BUFM is 28 , which falls in the worst performance range of the results associated with PROM. To summarize, PROM generates a solution that performs best in the three models while hedging against all kinds of real-life disruptions. Its solution has also provided a certain level of stability in performance in all scenarios with all levels of traffic densities since positive performance indicators are always obtained.

The simulation model estimates the total number of conflicts composed of three types of conflicts. It is necessary to identify the origins of the conflicts so as to better characterize the effects of perturbation in terms of conflict distribution in the network. In Fig. 10, four subfigures associated with four scenarios are displayed. In each figure, the link, node and runway conflicts are represented by stacked bar graphs based on the average conflict number of that specific type. The average number of conflicts of each type shows similar variation trend to the total number of conflicts over time. Another observation is that the link conflicts account for the majority in the total conflicts. The reason for fewer node conflicts can be concluded 


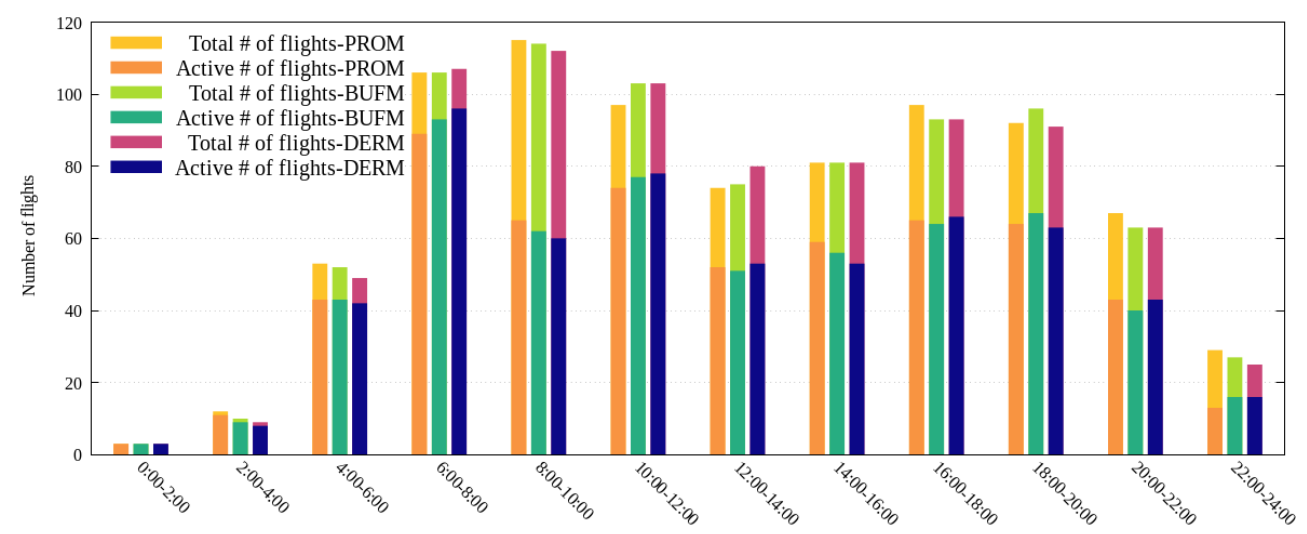

Figure 9: Number of active flights and total flights involved in each window in the simulation of the three models.

from two key aspects. First, the conflict detection for a link and the entry node of this link are based on same time information, but in some cases, the time separation requirements computed for node conflicts are smaller than those of the link conflicts. (see Eq. 2 and Eq. 3). Second, the number of nodes considered in the conflict evaluation is less than the number of links, because four node entry points are excluded. In the network, the runway separation violations have the least chance of occurring, since only two runways are involved. Fig. 10 highlights the advantage of PROM over BUFM and DERM.

\section{Conclusion}

This paper addresses the arrival aircraft scheduling problem under uncertainty by performing conflict detection and resolution for aircraft in a predefined network. A novel probabilistic model is proposed based on the deterministic model in 42. The uncertainty is managed by representing the time information used for conflict detection as random variables. Considering the uncertainty propagation in reality, the probability distributions of the random variables are characterized based on the Markov assumption for a flight during the operation in the TMA. By assuming that the prediction error is normally distributed, we benefit from the properties of the normal distribution to produce an efficient way of evaluating the probability of conflict occurrence between each two successively operated aircraft at specific locations. The objective function is formulated as the expected number of conflicts of all flights in the network and it can be analytically computed. Using this design, the computational complexity is reduced compared to the typical uncertainty optimization approaches. In our study, the uncertainty has been considered as an integrated term, therefore a wide application of this approach is possible.

The time decomposition and sliding window frame combined with simulated annealing has been proven to be efficient as a solution algorithm. For the proposed model, the sliding window frame also plays an important role in limiting the variance of the random variables in a reasonable range.

The work has been enriched by introducing a Monte-Carlo simulation framework with different scenarios representing the variability of the uncertainty elements. Except for the proposed model, DERM and BUFM represent the deterministic model without considering the uncertainty, and the model incorporating separation buffers are presented for the purpose of comparison. The solutions of three models are evaluated by means of stochastic perturbation for 12 windows with different traffic densities. Statistical analyses are conducted by comparing the conflicts generated in different scenarios in the simulation. A relative comparison is conducted by using the simulation results associated with the deterministic model as the basis upon which the conflict absorption percentages for the other two models are computed. Numerical results show the limitation of BUFM, as in some cases, it performs even worse than DERM. In contrast, the proposed model provides a certain level of stability as its solution can still absorb $25 \%$ of the conflicts with respect to the deterministic model under a case of high traffic density and high variance of perturbation. Moreover, 


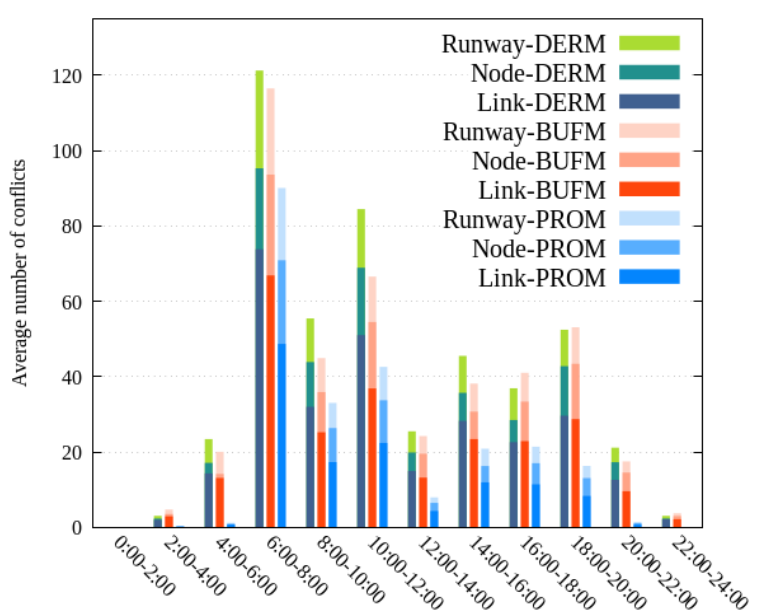

(a) Scenario N1.

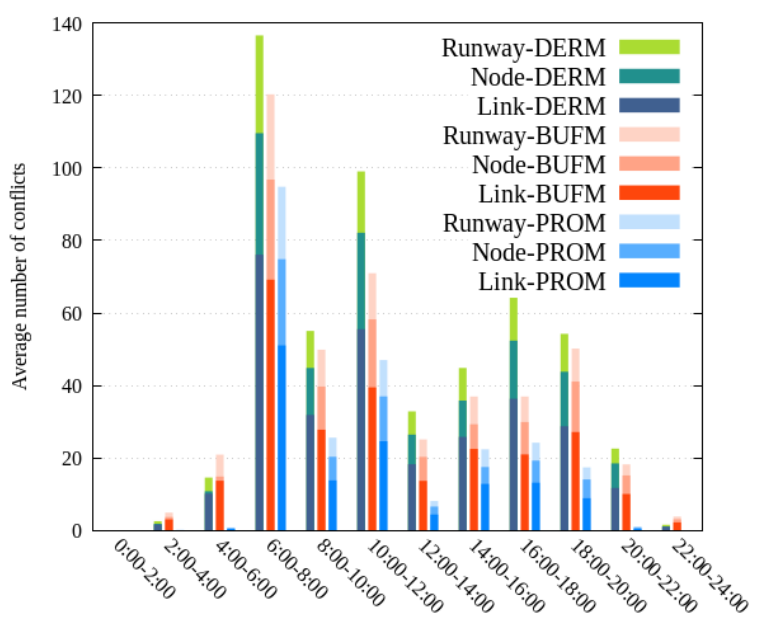

(c) Scenario U1.

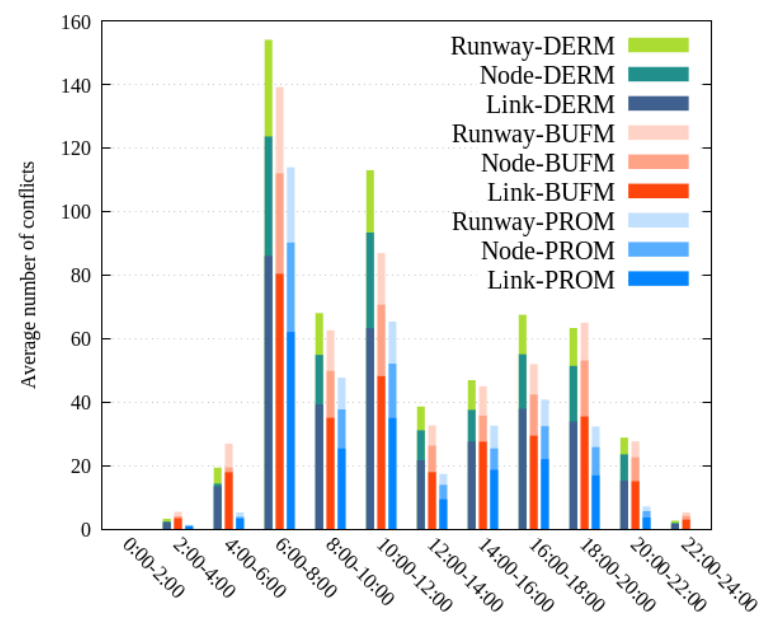

(b) Scenario N2.

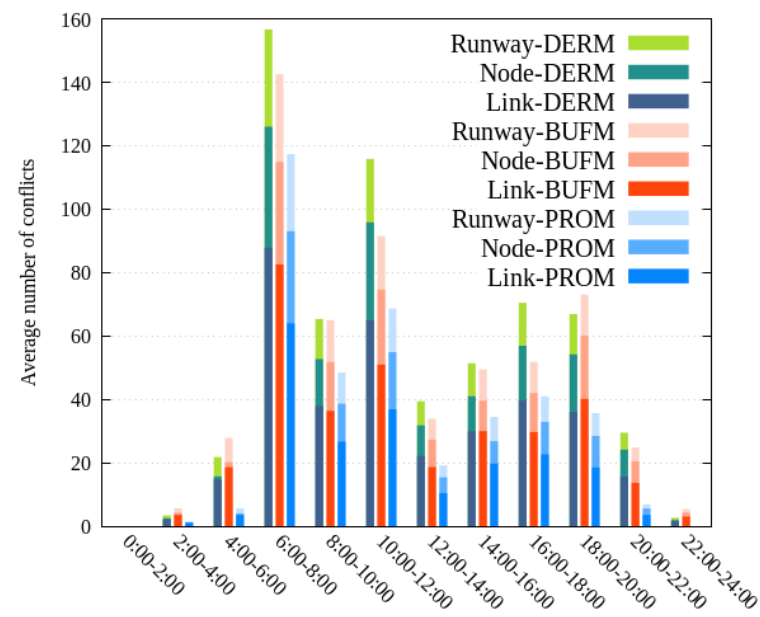

(d) Scenario U2.

Figure 10: Average number of conflicts comparison on links, nodes and runways of all the time windows based on four scenarios for the three models.

the type of the distributions of the perturbation does not cause a notable difference in the performance of conflict absorption.

Compared to other uncertainty optimization approaches, the proposed method has advantages given different perspectives. For example, robust optimization attempts to seek an optimal solution under the worst-case scenario which leads to a conservative result. This conservatism will lead to a waste of available time slots in arrival management, thus reducing efficiency. However, the proposed approach does not apply hard constraints, so that the balance between the available resources and current traffic density can be achieved while considering the robustness for the final result. Though belonging to the stochastic problem, in the proposed approach, the assumption of normality of the random variables eases the computational burden of using scenario-based solution approach (e.g. SAA). Therefore, it can have a more broadly application.

For future work, two directions can be considered. First, from the perspective of uncertainty optimization, the distributionally robust optimization emphasizes the ambiguity of the probability distribution of the uncertain parameter. Analogous to robust optimization that considers the worst-case parameter realization in the uncertainty set, distributionally robust optimization seeks to protect against the worst-case with 
ambiguous probability distribution. Under this case, the probability distribution for the uncertain parameter is unknown but belongs to an infinite set of predefined probability distributions [50. Distributionally robust optimization therefore offers a very interesting alternative to deal with the problem under uncertainty and indicates potential direction for future study. Second, it is important to consider the interests of other stakeholders, such as the delay time, fuel cost etc. In addition, the work can be extended to a broader range such as the enroute segment to be fully compatible with the Enhanced E-AMAN conception. Another possibility is to investigate the integrated optimization problem by taking into account the resources within the airport.

\section{Acknowledgements}

This research is supported by grants from the Funds of China Scholarship Council (201708070018). The authors would like to thank Serge Roux for his technical support. The authors would also like to thank the anonymous referees whose detailed comments have helped to improve the presentation of the paper.

\section{Appendix A. Proof of Lemmas}

In this appendix we prove Lemmas 1 and 2 We will use the following assumption, which is satisfied in our study case.

Assumption 1. For any two distinct adjacent links $\mu_{1}=\left(\nu_{1}, \nu\right)$ and $\mu_{2}=\left(\nu_{2}, \nu\right)$ (or $\left.\mu_{2}=\left(\nu, \nu_{2}\right)\right)$, the distance from $\nu_{2}$ to the link $\mu_{1}$ and from $\nu_{1}$ to $\mu_{2}$ are greater than or equal to $h$. By the distance from a point to a link, we mean the length of the shortest line joining the point to a point on the link.

\section{Appendix A.1. Proof of Lemma 1}

To shorten the notation let us denote the speed of aircraft $f$ by $v_{1}$ instead of $v_{f, \nu}$ and the speed of aircraft $g$ by $v_{2}$ instead of $v_{g, \nu}$. We assume that aircraft $g$ pass the node $\nu$ after $\delta$ units of time when $f$ passed it, that is $\delta=t_{g, \nu}-t_{f, \nu}$. Consider first the case where both aircraft enter node $\nu$ through the same link $\mu^{-}$ of length $l^{-}$then fly along the link $\mu^{+}$of length $l^{+}$(see Fig. 2a). We are interested in the occurrence of a node conflict at $\nu$ i.e. when $f$ flies on $\mu^{+}$and $g$ flies on $\mu^{-}$. If $\delta>\frac{l^{-}}{v_{2}}+\frac{l^{+}}{v_{1}}$, that is to say, if aircraft $f$ has left link $\mu^{+}$before aircraft $g$ enters the link $\mu^{-}$, then the aircraft are not in conflict. Hence we can assume that $\delta \leq \frac{l^{-}}{v_{2}}+\frac{l^{+}}{v_{1}}$. Under this condition, the (square of) distance between the two aircraft after $t$ units of time when aircraft $f$ passed the node $\nu$ is given by:

$$
d^{2}(t, \delta)=\left(t\left(v_{1} \cos \theta-v_{2}\right)+\delta v_{2}\right)^{2}+t^{2} v_{1}^{2} \sin ^{2} \theta
$$

which has to be considered only during the presence of the two aircraft on their respective links, that is $t \in\left[\max \left(0, \delta-\frac{l^{-}}{v_{2}}\right), \min \left(\frac{l^{+}}{v_{1}}, \delta\right)\right]$. Let us define the function $m$ representing the minimum (squared) distance between the two aircraft during this period, or more precisely

$$
m(\delta):=\min _{t \in\left[\max \left(0, \delta-\frac{l^{-}}{v_{2}}\right), \min \left(\frac{l^{+}}{v_{1}}, \delta\right)\right]} d^{2}(t, \delta) .
$$

We have

$$
\begin{aligned}
\frac{\partial d^{2}}{\partial t}(t, \delta) & =2\left(v_{1} \cos \theta-v_{2}\right)\left(t\left(v_{1} \cos \theta-v_{2}\right)+\delta v_{2}\right)+2 t v_{1}^{2} \sin ^{2} \theta \\
& =2 \delta v_{2}\left(v_{1} \cos \theta-v_{2}\right)+2 t\left(v_{1}^{2}+v_{2}^{2}-2 v_{1} v_{2} \cos \theta\right)
\end{aligned}
$$

so the second order polynomial with a positive leading coefficient $d^{2}(\delta, \cdot)$ achieves its minimum on $\mathbb{R}$ at $t^{*}(\delta)=\alpha \delta$, where $\alpha=\frac{v_{2}\left(v_{2}-v_{1} \cos \theta\right)}{v_{1}^{2}+v_{2}^{2}-2 v_{1} v_{2} \cos \theta}$. The following three exclusive cases will be considered successively: 
$\alpha \leq 0, \alpha \geq 1$ and $0<\alpha<1$. These cases respectively correspond to the following cases in the definition of the expression of $a$ in Lemma 1:

1. $v_{2} \leq v_{1} \cos \theta$

2. $v_{1} \leq v_{2} \cos \theta$

3. $v_{2}>v_{1} \cos \theta$ and $v_{1}>v_{2} \cos \theta$

In the first case $\left(v_{2} \leq v_{1} \cos \theta\right)$, we have $\max \left(0, \delta-\frac{l^{-}}{v_{2}}\right) \geq t^{*}(\delta)$. The minimum A.1 is then achieved at $\max \left(0, \delta-\frac{l^{-}}{v_{2}}\right)$ with a value

$$
m^{\frac{1}{2}}(\delta)= \begin{cases}\delta v_{2} & \text { if } \delta \leq \frac{l^{-}}{v_{2}} \\ \geq h, & \text { otherwise }\end{cases}
$$

The last inequality follows from the fact that at $t=\delta-\frac{l^{-}}{v_{2}}$, the aircraft $g$ is at the entry node of link $\mu^{-}$ and Assumption 1. Consequently $m^{\frac{1}{2}}(\delta) \geq h$ if and only if $\delta \geq \frac{h}{v_{2}}$.

In the second case $\left(v_{1} \leq v_{2} \cos \theta\right), t^{*}(\delta) \geq \min \left(\delta, \frac{l^{+}}{v_{1}}\right)$. The minimum A.1] is achieved at $\min \left(\delta, \frac{l^{+}}{v_{1}}\right)$ with a value

$$
m^{\frac{1}{2}}(\delta)= \begin{cases}\delta v_{1} & \text { if } \delta \leq \frac{l^{+}}{v_{1}} \\ \geq h, & \text { otherwise }\end{cases}
$$

The last inequality follows from the fact that at $t=\frac{l^{+}}{v_{1}}$ the aircraft $f$ is at the exit node of link $\mu^{+}$and Assumption 1. Consequently $m^{\frac{1}{2}}(\delta) \geq h$ if and only if $\delta \geq \frac{h}{v_{1}}$.

In the third case $\left(v_{2}>v_{1} \cos \theta\right.$ and $\left.v_{1}>v_{2} \cos \theta\right)$, we have

- If $\delta \in\left[0, \min \left(\frac{l^{-}}{(1-\alpha) v_{2}}, \frac{l^{+}}{\alpha v_{1}}\right)\right]$, then $t^{*}(\delta)=\alpha \delta \in\left[\max \left(0, \delta-\frac{l^{-}}{v_{2}}\right), \min \left(\frac{l^{+}}{v_{1}}, \delta\right)\right]$ and consequently we have $m^{\frac{1}{2}}(\delta)=d(\alpha \delta, \delta)=\frac{\delta v_{1} v_{2} \sin \theta}{\sqrt{v_{1}^{2}+v_{2}^{2}-2 v_{1} v_{2} \cos \theta}}$.

- If $\delta>\min \left(\frac{l^{-}}{(1-\alpha) v_{2}}, \frac{l^{+}}{\alpha v_{1}}\right)$ we consider two sub-cases : either the last min equals to $\frac{l^{+}}{\alpha v_{1}}$ or to $\frac{l^{-}}{(1-\alpha) v_{2}}$. In the first sub-case, $\delta>\frac{l^{+}}{\alpha v_{1}}$ implies $\delta>\alpha \delta>\frac{l^{+}}{v_{1}}$, so the minimum A.1 is achieved at $\frac{l^{+}}{v_{1}}$ when the aircraft $f$ is at the exit node of link $\mu^{+}$, and thanks to Assumption $1, m^{\frac{1}{2}}(\delta) \geq h$. In the other sub-case, $\delta>\frac{l^{-}}{(1-\alpha) v_{2}}$ implies $\delta-\frac{l^{-}}{v_{2}}>\alpha \delta>0$, so the minimum A.1 is achieved at $\delta-\frac{l^{-}}{v_{2}}$ when the aircraft $g$ is at the entry node of link $\mu^{-}$, and again thanks to Assumption $1 . m^{\frac{1}{2}}(\delta) \geq h$.

In sum, we have

$$
m^{\frac{1}{2}}(\delta)= \begin{cases}d(\alpha \delta, \delta)=\frac{\delta v_{1} v_{2} \sin \theta}{\sqrt{v_{1}^{2}+v_{2}^{2}-2 v_{1} v_{2} \cos \theta}} & \text { if } \delta \in\left[0, \min \left(\frac{l^{-}}{(1-\alpha) v_{2}}, \frac{l^{+}}{\alpha v_{1}}\right)\right] \\ \geq h, & \text { otherwise. }\end{cases}
$$

Consequently $m^{\frac{1}{2}}(\delta) \geq h$ if and only if $\delta \geq \frac{h \sqrt{v_{1}^{2}+v_{2}^{2}-2 v_{1} v_{2} \cos \theta}}{v_{1} v_{2} \sin \theta}$.

Gathering all the cases we obtain, $m^{\frac{1}{2}}(\delta) \geq h$, meaning that no horizontal conflict occur between $f$ and $g$ at node $\nu$, if and only if $\delta \geq a$, where $a$ is defined by

$$
a= \begin{cases}\frac{h}{v_{2}} & \text { if } v_{2} \leq v_{1} \cos \theta \\ \frac{h}{v_{1}} & \text { if } v_{1} \leq v_{2} \cos \theta \\ \frac{h \sqrt{v_{1}^{2}+v_{2}^{2}-2 v_{1} v_{2} \cos \theta}}{v_{1} v_{2} \sin \theta}, & \text { otherwise. }\end{cases}
$$

Considering now the case where aircraft $f$ and $g$ enter node $\nu$ from different links $\mu_{1}^{-}$and $\mu_{2}^{-}$then fly along a link $\mu^{+}$(see Fig. 2b). A node conflict can happen in one of the two following situations:

- aircraft $f$ fly on $\mu^{+}$and aircraft $g$ on link $\mu_{2}^{-}$, 
- aircraft $f$ fly on $\mu_{1}^{-}$and aircraft $g$ on link $\mu_{2}^{-}$.

From the above, no conflict can happen in the first situation if and only if $\delta \geq a$, Consider now the second situation. Let us denote by $l_{i}$ the length of link $\mu_{i}^{-}, i=1,2$. If $\delta>\min \left(\frac{l_{1}}{v_{1}}, \frac{l_{2}}{v_{2}}\right)$, that is to say, if aircraft $f$ leaves link $\mu_{1}^{-}$before aircraft $g$ enters the link $\mu_{2}^{-}$, then the aircraft are not in conflict. Hence, we assume that $\delta \leq \min \left(\frac{l_{1}}{v_{1}}, \frac{l_{2}}{v_{2}}\right)$.

Under this condition, the (square of) distance between the two aircraft $t$ units of time before aircraft $f$ passes node $\nu$ is given by:

$$
d^{2}(t, \delta)=\left(t\left(v_{2}-v_{1} \cos \varphi\right)+\delta v_{2}\right)^{2}+t^{2} v_{1}^{2} \sin ^{2} \varphi,
$$

which has to be considered only during the presence of the two aircraft on their respective links, that is $t \in\left[0, \min \left(\frac{l_{1}}{v_{1}}, \frac{l_{2}}{v_{2}}-\delta\right)\right]$. By arguments similar to the first part of the proof, we can establish that $m(\delta):=$ $\min _{t \in\left[0, \min \left(\frac{l_{1}}{v_{1}}, \frac{l_{2}}{v_{2}}-\delta\right)\right]} d^{2}(t, \delta) \geq h^{2}$ if and only if $\delta \geq b:=\left\{\begin{array}{ll}\frac{h}{v_{2}} & \text { if } v_{1} \cos \varphi \leq v_{2}, \\ \frac{h \sqrt{v_{1}^{2}+v_{2}^{2}-2 v_{1} v_{2} \cos \varphi}}{v_{1} v_{2} \sin \varphi}, & \text { otherwise. }\end{array}\right.$.

In summary, putting the two situations together implies that the absence of conflict is equivalent to $\delta \geq \max (a, b)$.

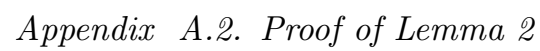

We remark that if $\delta>\frac{l}{v_{1}}$, that is to say, if aircraft $f$ leaves link $\mu$ before aircraft $g$ enters it, then the aircraft are not in conflict. Hence, assuming that $\delta \leq \frac{l}{v_{1}}$, the distance between the two aircraft after $t$ units of time that aircraft $g$ has passed node $\nu$ is given by:

$$
d(t, \delta)=(\delta+t) v_{1}-t v_{2}
$$

Then, there is no conflict between $f$ and $g$ at link $\mu$ if and only if the wake turbulence separation is satisfied at the entry (at $t=0)$ and the exit (at $t=\frac{l}{v_{1}}-\delta$ ) of link $\mu$ :

$$
\delta v_{1} \geq s_{f, g} \text { and } l-v_{2}\left(\frac{l}{v_{1}}-\delta\right) \geq s_{f, g}
$$

or equivalently

$$
\delta \geq \max \left(\frac{s_{f, g}}{v_{1}}, \frac{s_{f, g}}{v_{2}}+l \frac{v_{2}-v_{1}}{v_{1} v_{2}}\right)
$$

\section{References}

[1] ICAO, Effects of Novel Coronavirus (COVID-19) on Civil Aviation:Economic Impact Analysis, https://www.icao.int/ sustainability/Documents/Covid-19/ICAO_coronavirus_Econ_Impact.pdf, accessed: Jan-2021 (2021).

[2] EUROCONTROL, Eurocontrol revises downwards draft traffic scenarios for september to february 2021, "https: //www. eurocontrol.int/sites/default/files/2020-09/eurocontrol-draft-traffic-scenarios-14092020.pdf", accessed: 14-Sep-2020 (2020).

[3] S. V. Gudmundsson, M. Cattaneo, R. Redondi, Forecasting temporal world recovery in air transport markets in the presence of large economic shocks: The case of covid-19, Journal of Air Transport Management 91 (2021) 102007.

[4] EUROCONTROL, EUROCONTROL Five-Year Forecast 2020-2024, https://www.eurocontrol.int/publication/ eurocontrol-five-year-forecast-2020-2024, accessed: Nov-2020 (2020).

[5] SESAR, Sesar 2020 multi-annual work programme, SESAR Joint Undertaking, Brussels, Belgium (2015) 565.

[6] SESAR, Where to start in building resilience in air traffic management, "https://www.sesarju.eu/news/ where-start-building-resilience-air-traffic-management", accessed: 26-May-2020 (2020).

[7] J. E. Beasley, M. Krishnamoorthy, Y. M. Sharaiha, D. Abramson, Scheduling aircraft landings-the static case, Transportation science 34 (2) (2000) 180-197.

[8] J. E. Beasley, J. Sonander, P. Havelock, Scheduling aircraft landings at london heathrow using a population heuristic, Journal of the operational Research Society 52 (5) (2001) 483-493.

[9] R. G. Dear, The dynamic scheduling of aircraft in the near terminal area, Tech. rep., Cambridge, Mass.: Flight Transportation Laboratory, Massachusetts Institute (1976). 
[10] J. E. Beasley, M. Krishnamoorthy, Y. M. Sharaiha, D. Abramson, Displacement problem and dynamically scheduling aircraft landings, Journal of the operational research society 55 (1) (2004) 54-64.

[11] H. Balakrishnan, B. Chandran, Scheduling aircraft landings under constrained position shifting, in: AIAA guidance, navigation, and control conference and exhibit, 2006, p. 6320.

[12] H. Lee, H. Balakrishnan, A study of tradeoffs in scheduling terminal-area operations, Proceedings of the IEEE 96 (12) (2008) 2081-2095.

[13] A. Mascis, D. Pacciarelli, Job-shop scheduling with blocking and no-wait constraints, European Journal of Operational Research 143 (3) (2002) 498-517.

[14] L. Bianco, P. Dell'Olmo, S. Giordani, Scheduling models for air traffic control in terminal areas, Journal of Scheduling 9 (3) (2006) 223-253.

15] M. Sama, A. D'Ariano, P. D'Ariano, D. Pacciarelli, Optimal aircraft scheduling and routing at a terminal control area during disturbances, Transportation Research Part C: Emerging Technologies 47 (2014) 61-85.

[16] L. Boursier, B. Favennec, E. Hoffman, A. Trzmiel, F. Vergne, K. Zeghal, Merging arrival flows without heading instructions, in: 7th USA/Europe air traffic management R\&D seminar, 2007, pp. 1-8.

[17] O. S. Meric, O. Turan, Evaluation of aircraft descent profile, Energy Procedia 95 (2016) 308-313.

[18] M. Liang, D. Delahaye, P. Marechal, Conflict-free arrival and departure trajectory planning for parallel runway with advanced point-merge system, Transportation Research Part C: Emerging Technologies 95 (2018) 207-227.

[19] M. Tielrooij, C. Borst, M. M. Van Paassen, M. Mulder, Predicting arrival time uncertainty from actual flight information, in: Proceedings of the 11th USA/Europe Air Traffic Management Research and Development Seminar, 2015, pp. 577-586.

[20] C. Wanke, S. Mulgund, D. Greenbaum, L. Song, Modeling traffic prediction uncertainty for traffic management decision support, in: AIAA Guidance, Navigation, and Control Conference and Exhibit, 2004, p. 5230.

[21] J. Mercer, N. Bienert, A. Gomez, S. Hunt, J. M. Kraut, L. Martin, S. Morey, S. Green, T. Prevôt, M. G. Wu, The impact of trajectory prediction uncertainty on air traffic controller performance and acceptability, in: 2013 Aviation Technology, Integration, and Operations Conference, 2013, p. 4320.

[22] Z. Li, M. Ierapetritou, Process scheduling under uncertainty: Review and challenges, Computers \& Chemical Engineering $32(4-5)(2008) 715-727$.

[23] N. V. Sahinidis, Optimization under uncertainty: state-of-the-art and opportunities, Computers \& Chemical Engineering 28 (6-7) (2004) 971-983.

[24] C. Bosson, M. Xue, S. Zelinski, Optimizing integrated terminal airspace operations under uncertainty, in: 2014 IEEE/AIAA 33rd Digital Avionics Systems Conference (DASC), IEEE, 2014, pp. 1A3-1.

[25] A. Khassiba, F. Bastin, B. Gendron, S. Cafieri, M. Mongeau, Extended aircraft arrival management under uncertainty: A computational study, Journal of Air Transportation 27 (3) (2019) 131-143.

[26] A. J. Kleywegt, A. Shapiro, T. Homem-de Mello, The sample average approximation method for stochastic discrete optimization, SIAM Journal on Optimization 12 (2) (2002) 479-502.

[27] A. Ben-Tal, A. Nemirovski, Robust optimization-methodology and applications, Mathematical programming 92 (3) (2002) 453-480.

[28] D. Bertsimas, M. Sim, Robust discrete optimization and network flows, Mathematical programming 98 (1-3) (2003) 49-71.

[29] K. Ng, C. Lee, F. T. Chan, Y. Qin, Robust aircraft sequencing and scheduling problem with arrival/departure delay using the min-max regret approach, Transportation Research Part E: Logistics and Transportation Review 106 (2017) 115-136.

[30] M. Kapolke, N. Fürstenau, A. Heidt, F. Liers, M. Mittendorf, C. Weiß, Pre-tactical optimization of runway utilization under uncertainty, Journal of Air Transport Management 56 (2016) 48-56.

[31] L. Marla, A. Rikun, G. Stauffer, E. Pratsini, Robust modeling and planning: Insights from three industrial applications, Operations Research Perspectives 7 (2020) 100150

[32] E. Esche, B. You, J.-U. Repke, Optimal design via chance-constrained or two-stage stochastic programming, in: Computer Aided Chemical Engineering, Vol. 47, Elsevier, 2019, pp. 169-174.

[33] A. Charnes, W. W. Cooper, Chance-constrained programming, Management science 6 (1) (1959) 73-79.

[34] J. Chen, L. Chen, D. Sun, Air traffic flow management under uncertainty using chance-constrained optimization, Transportation Research Part B: Methodological 102 (2017) 124-141.

[35] E. P. Gilbo, S. B. Smith, et al., New method for probabilistic traffic demand predictions for en route sectors based on uncertain predictions of individual flight events.

[36] G. M. Caron, P. Savéant, M. Schoenauer, Multiobjective tactical planning under uncertainty for air traffic flow and capacity management, in: 2013 IEEE Congress on Evolutionary Computation, IEEE, 2013, pp. 1548-1555.

[37] F. Gonze, E. Huens, R. M. Jungers, A. Simonetto, J. Boucquey, Probabilistic occupancy counts and flight criticality measures in air traffic management, Journal of Air Transportation 26 (3) (2018) 94-103.

[38] L. Meyn, H. Erzberger, Airport arrival capacity benefits due to improved scheduling accuracy, in: AIAA 5th ATIO and16th Lighter-Than-Air Sys Tech. and Balloon Systems Conferences, 2005, p. 7376.

[39] A. Heidt, H. Helmke, M. Kapolke, F. Liers, A. Martin, Robust runway scheduling under uncertain conditions, Journal of Air Transport Management 56 (2016) 28-37.

[40] P. Scala, M. M. Mota, J. Ma, D. Delahaye, Tackling uncertainty for the development of efficient decision support system in air traffic management, IEEE Transactions on Intelligent Transportation Systems 21 (8) (2019) 3233-3246.

[41] P. Scala, M. M. Mota, C.-L. Wu, D. Delahaye, An optimization-simulation closed-loop feedback framework for modeling the airport capacity management problem under uncertainty, Transportation Research Part C: Emerging Technologies $124(2021) 102937$.

[42] J. Ma, D. Delahaye, M. Sbihi, P. Scala, M. A. M. Mota, Integrated optimization of terminal maneuvering area and airport at the macroscopic level, Transportation Research Part C: Emerging Technologies 98 (2019) 338-357. 
[43] M. Bagamanova, M. M. Mota, Reducing airport environmental footprint using a disruption-aware stand assignment approach, Transportation Research Part D: Transport and Environment 89 (2020) 102634.

[44] J. Ma, D. Delahaye, M. Sbihi, M. Mongeau, Merging flows in terminal moneuvering area using time decomposition approach, 2016.

[45] M. J. Frankovich, Air traffic flow management at airports: A unified optimization approach, Ph.D. thesis, Massachusetts Institute of Technology (2012).

[46] A. Khassiba, F. Bastin, S. Cafieri, B. Gendron, M. Mongeau, Two-stage stochastic mixed-integer programming with chance constraints for extended aircraft arrival management, Transportation Science 54 (4) (2020) 897-919.

[47] G. Marceau Caron, Optimization and uncertainty handling in air traffic management, Ph.D. thesis, Paris 11 (2014).

[48] W. D. Cottrell, Simplified program evaluation and review technique (pert), Journal of construction Engineering and Management 125 (1) (1999) 16-22.

[49] P. J. Van Laarhoven, E. H. Aarts, Simulated annealing, in: Simulated annealing: Theory and applications, Springer, 1987, pp. 7-15.

[50] H. Rahimian, S. Mehrotra, Distributionally robust optimization: A review, arXiv preprint arXiv:1908.05659. 